\title{
Mycobacterium tuberculosis Heat-Shock Protein 16.3 Induces Macrophage M2 Polarization Through CCRL2/CX3CR1
}

\author{
Yanhao Zhang, ${ }^{1}$ Shanshan Li, ${ }^{1}$ Qianyi Liu, ${ }^{1}$ Ruiying Long, ${ }^{1}$ Jihong Feng, ${ }^{1}$ Huan Qin, ${ }^{1}$ Mao Li, ${ }^{1}$ \\ Liping Liu, ${ }^{1}$ and Junmin Luo ${ }^{1,2}$
}

\begin{abstract}
Mycobacterium tuberculosis, the pathogen of tuberculosis (TB), can survive in host macrophages and induce macrophages to M2 phenotype might result in latent MTB infection. During the latent phase, the expression of MTB heat-shock protein 16.3 (Hsp16.3) is markedly increased among most of bacterial proteins, but the role of Hsp16.3 in macrophage M2 polarization is not clear. In this work, we found that macrophages incubated with 100 ng/ml MTB Hsp16.3 increased the production of Arg-1, IL-10, TGF-beta, and CD206. These results showed that MTB Hsp16.3 may induce macrophage M2 phenotype. And the interaction of Hsp16.3 with macrophages was found to depend on chemokine receptors CCRL2 and CX3CR1. Additionally, we used overexpression and silencing techniques to further verify the effect of CCRL2 and CX3CR1 on MTB Hsp16.3-induced M2 polarization macrophages. Furthermore, we explored the downstream signaling molecules of CCRL2 and CX3CR1 and we found MTB Hsp16.3 altered the signal transduction of the AKT/ERK/p38-MAPK. Taken together, this study provides evidence that MTB Hsp16.3 promotes macrophages to M2 phenotype and explores its underlying mechanism.
\end{abstract}

KEY WORDS: Mycobacterium tuberculosis; macrophage polarization; Mycobacterium tuberculosis heat-shock protein 16.3; chemokine receptors.

\section{INTRODUCTION}

Tuberculosis (TB) poses a serious threat to human health. According to the WHO, the number of new TB cases in 2017 was approximately 10 million, and the global death rate is approximately 1.57 million [1].

Yanhao Zhang and Shanshan Li contributed equally to this work.

${ }^{1}$ Department of Immunology, Zunyi Medical University, No. 6, Xuefu West Road, Xinpu District, Zunyi, 563000, Guizhou, China

${ }^{2}$ To whom correspondence should be addressed at Department of Immunology, Zunyi Medical University, No. 6, Xuefu West Road, Xinpu District, Zunyi, 563000, Guizhou, China. E-mail: Luojmktz@163.com
Mycobacterium tuberculosis (MTB), an intracellular pathogen, causes TB and leads to both latent and acute infections. MTB usually enters the body through inhalation into the lungs, and alveolar macrophages phagocytose the bacteria and move to the pulmonary interstitial space where they recruit mononuclear-derived macrophages and other immune cells to form tuberculous granuloma. It is generally considered that the main function of the tuberculous granuloma is to localize the infecting bacteria and prevent the spread of infection [2], but MTB uses this opportunity to inhabit macrophages and survive for a prolonged time through their own components and virulence factors [3]. 
Macrophages that differentiate from monocytes and bone marrow hematopoietic stem cells are the first line of defense against microbes; they have cytokine secretion, phagocytosis, and antigen presentation functions. Macrophages exhibit vast functional plasticity and polarize to a certain phenotype in response to the microenvironment. Due to their functional characteristics and morphology, macrophages can be classified into classically activated macrophages (or M1) and alternatively activated macrophages (or M2). M1 macrophages, polarized by interferongamma (IFN- $\gamma$ ) and lipopolysaccharide (LPS), have increased expression levels of inflammatory cytokines (interleukin (IL)-12, tumor necrosis factor- $\alpha$ (TNF- $\alpha$ ), and IL-6) [4], inducible nitric oxide synthase (iNOS), and CD86, and have the capacity to kill pathogens and tumor cells, and promote a Th1-type immune response.

In contrast, M2 macrophages are associated with antiinflammatory activity. According to different stimuli, M2 phenotype macrophages can be further divided into three subpopulations, M2a, M2b, and M2c. M2a macrophages induced by IL- 4 and IL-13 express the mannose receptor, scavenger receptor, and arginase-1 (Arg-1); M2b macrophages, stimulated by IL-1 receptor ligands and immune complexes, increase the production of IL-10 and promote the Th2 response, whereas M2c macrophages, mainly induced by IL-10, secrete pentraxin 3 and chitinase 3-like 3 and play a role in wound healing [5].

Research has shown that macrophages play a dual role in MTB infection. On the one hand, macrophages are the main immune effector cells and antigen-presenting cells responsible for the anti-tuberculosis response; on the other hand, macrophages are a habitat of M. tuberculosis, and they provide protection to MTB [6]. MTB has evolved several strategies to reside and even replicate within macrophages, including preventing phagosome-lysosome fusion, inhibiting phagolysosomal maturation, and inducing M2 macrophage polarization [2]. A study showed that M2 phenotype macrophages contribute to the creation of a suppressive microenvironment that can promote MTB intracellular growth and latent infection. Therefore, an improved understanding of macrophage polarization during MTB infection may be helpful to cure TB.

Recent studies have found that small heat-shock proteins (sHsps) play an important role in the development of tuberculosis. sHsps, which maintain cell stability, aid denatured protein refolding, and participate in cell damage and repair, are present in most organisms. MTB heat-shock protein 16.3 (Hsp16.3), a member of the $\alpha$-crystal superfamily, is encoded by the HspX gene and is known as Acr1, 16-kDa antigen, and Rv2031c. Hsp16.3 has a relative molecular weight of approximately $16.3 \mathrm{kDa}$. MTB invades the body and is phagocytosed by pulmonary macrophages; in order for MTB to survive in the macrophage environment, DosR gene expression is activated, which upregulates the expression of many bacterial proteins, of which MTB Hsp16.3 is the highest [7, 8]. Studies have shown that MTB Hsp16.3 impacts macrophage apoptosis and autophagy [9]. All of the above studies suggest that MTB Hsp16.3 plays a role in the survival of host macrophages during latent infection.

In our previous work, we constructed a prokaryotic expression vector, pET28a-MTB Hsp16.3, which induces the expression of MTB Hsp16.3 and purified the fusion protein through nickel affinity chromatography [10]. We found that MTB Hsp16.3 can induce M2 phenotype polarization in RAW264.7 cells. Incubation of macrophages with Hsp16.3 recombinant protein increases the secretion of TGF- $\beta$ and IL-10, and compared with untreated macrophages; treated macrophages became long and stringy with increased numbers of pseudopods. MTB Hsp16.3 can induce the polarization of macrophages to the M2 phenotype, but the mechanism by which Hsp16.3 regulates macrophage polarization remains to be elucidated. In this study, we found that Hsp16.3 can induce the bone marrowderived macrophage (BMDM) M2-like phenotype via CCRL2/CX3CR1 and may be mediated by the AKT/ ERK/p38-MAPK signaling pathway.

\section{MATERIALS AND METHODS}

\section{Animals}

Female BALB/c mice (6-8 weeks old) were from the Experimental Animals Center of Chongqing Medical University, China. All the animals were housed in specific pathogen-free environment with regular food and water.

\section{Plasmid Construction}

pcDNA3.1-CCRL2 (termed p-CCRL2) and pcDNA3.1-CX3CR1 (termed p-CX3CR1) plasmids were constructed. The CCRL2 and CX3CR1 genes were amplified by RT-PCR from the mRNA of macrophages derived from BALB/c mice (CCRL2: 5'-CGGGATCCATGGATAACTAC ACAGTGGCC C-3' (forward), 5'-CCCAAGCTTTTATA TTATATCCTGCCTTTGATGCA-3' (reverse). CX-3CR1: 5'-CGGGATCCATGTCCACCTCCTTCCCTG-3' (forward), 5'-CCCAAGCTTTCAGAGCAGGAGAGA CCCAT-3' (reverse). The eukaryotic expression vector pcDNA3.1-CCRL2 and pcDNA3.1-CX3CR1 products were 
digested with BamHI and HindIII, respectively. Positive clone identity was verified by plasmid DNA sequencing (Invitrogen, Shanghai, China). Endotoxin-free plasmids were obtained using an Endofree Plasmid Mega kit (QIAGEN $\mathrm{GmbH}$, Hilden, Germany). Then, plasmids were transiently transfected into the macrophages using Lipofectamine 2000 (Invitrogen, Shanghai, China) according to the manufacturer's instructions.

\section{Cell Culture and Transfection}

Bone marrow (BM) cells were isolated from the tibias and femurs of BALB/c mice, and the cells were cultured in Dulbecco's modified Eagle's medium (DMEM) low glucose (Gibco) supplemented with 10\% FBS and $20 \mathrm{ng} / \mathrm{ml}$ GM-CSF. Cells were harvested on day 7 for further experiments. BM cells were identified by flow cytometry with an anti-mouse CD11b (APC) antibody (cat. no. 17-011282, eBioscience) and an anti-mouse F4/80 (FITC) antibody (cat. no. 11-4801-82, eBioscience). Cells were cultured in a humidified atmosphere of $5 \% \mathrm{CO}_{2}$ at $37{ }^{\circ} \mathrm{C}$. In terms of cell transfection, BMDMs were inoculated at a density of $60 \sim 80 \%$ before the transfection experiment. After $24 \mathrm{~h}$, cells were transiently transfected with the indicated vectors with Lipofectamine 2000 according to the manufacturer's instructions. Then, the cells were harvested at different time points.

\section{RNAi Experiments}

Monolayers of cells cultured to approximately $70 \%$ confluency were subjected to siRNA transfection using the protocol recommended by the manufacturer (Ribort). Realtime PCR analysis was used to quantify the expression levels of siRNA-targeted genes.

\section{Real-Time PCR Assay}

Total RNA was extracted from the cells using TRIzol (TAKARA, Beijing, China) according to the manufacturer's instructions, and then total RNA was reverse transcribed to cDNA using a PrimeScript RT Master Mix kit (TAKARA, Beijing, China) following the manufacturer's instructions. The primers and protocol used for amplification were as follows:

GAPDH, 5'-GAGCCAAACGGGTCATCATCT-3' (forward), 5'-GAGGGGCCATCCACAGTC TT-3' (reverse); TNF- $\alpha$, 5'-CAGGGGCCACCACGCTCTTC-3' (forward), 5'-TTTGTGAGTGTGAGGGTCT GG-3' (reverse); IL-10, 5'-TACAGCCGGGAAGACAATAA-3' (forward), 5'-AGGAGTCGGTTAGCA GTATG-3' (reverse); TGF- $\beta$, 5'-GGCGGTGCTCGCTTTGTA-3' (forward), 5'-TCCCGAATGTCTGA CGTATTGA-3' (reverse); iNOS, 5'-CTGCAGCACTTGGATCAGGAACCTG-3' (forward), 5'-GGAGT AGCCTGTGTGTGCACCTGGA A-3' (reverse); Arg-1, 5'-CAGAAGAATGGAAG AGTCAG-3' (forward), 5'-CAGATATGCAGGGA GTCACC-3' (reverse); YM-1, 5'-GCAGAAGCTCTCCA ATCCTG-3' (forward), 5'-ATTGGCCTGTCCTT AGCCCAACTG-3' (reverse); Fizz1, 5'-GCTG ATGGTCCCAGTG AAAC-3' (forward), and 5'-CCAG TAGCAGTCATCCCAGC-3' (reverse). Real-time PCRs were performed in a BIO-RAD CFX96 detection system (Bio-Rad Laboratories) using a SYBR Premix Ex TaqTM kit (TAKARA, Beijing, China). The standard PCR conditions consisted of $95{ }^{\circ} \mathrm{C}$ for $30 \mathrm{~s}$, followed by 40 cycles of $95^{\circ} \mathrm{C}$ for $5 \mathrm{~s}$ and $60{ }^{\circ} \mathrm{C}$ for $34 \mathrm{~s}$, with a final dissociation stage. The relative expression of the indicated genes was calculated using the comparative threshold cycle $(\mathrm{Ct})$ method.

\section{Flow Cytometry}

BMDMs were stained with an anti-mouse F4/80 (FITC) antibody (cat. no. 11-4801-82, eBioscience), an anti-mouse CD206 (APC) antibody (cat. no. 17-2061-82, eBioscience), and an anti-mouse NOS2 (PE) antibody (cat. no. 25-5920-82, eBioscience) for $30 \mathrm{~min}$ at $4{ }^{\circ} \mathrm{C}$ and then washed with PBS and analyzed in a flow cytometer (Beckman Coulter, USA).

\section{ELISA}

Cell culture supernatant was collected, and then TNF$\alpha$, IL-10, and TGF- $\beta$ levels were determined by an ELISA kit (eBioscience, CA) according to the manufacturer's instructions. Standard curves of these cytokines were obtained using the recombinant standard proteins provided by the manufacturer.

\section{Western Blot Analyses}

Western blotting was performed on cytosolic cellular extracts. Cells were washed with cold phosphate-buffered saline and lysed for $15 \mathrm{~min}$ on ice in $0.5 \mathrm{ml}$ of lysis buffer containing protease and phosphatase inhibitors. Cell lysates were clarified by centrifugation $\left(4^{\circ} \mathrm{C}, 15 \mathrm{~min}, 12,000 \mathrm{rpm}\right)$, and protein was subjected to $10 \%$ sodium dodecyl sulfatePAGE (SDS-PAGE) and transferred to a nitrocellulose membrane using a wet transfer system. Membranes were incubated with 5\% skim milk dissolved in TBS plus $0.05 \%$ Tween 20 (TBST) for $1 \mathrm{~h}$ to block nonspecific protein- 

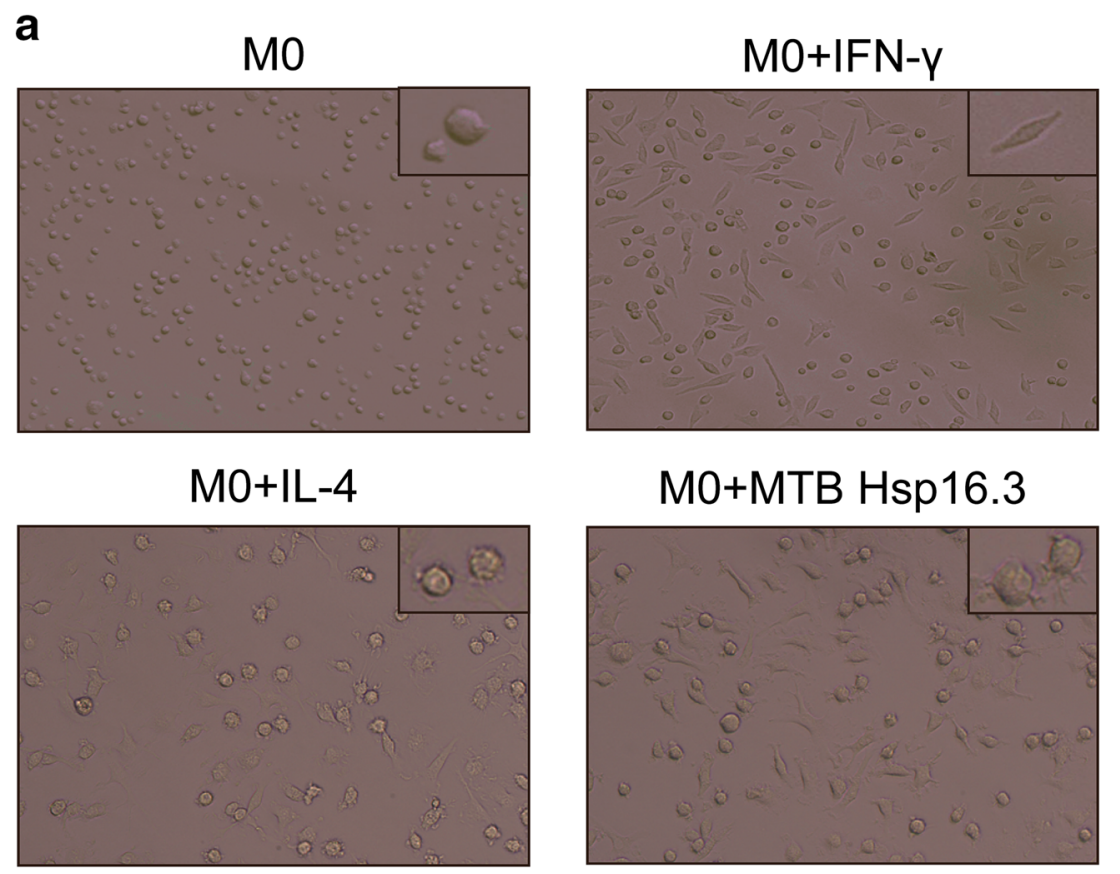

Fig. 1. MTB Hsp16.3 induces mouse bone marrow-derived macrophage (BMDM) M2 polarization. Bone marrow cells were isolated from the tibias and femurs of BALB/c mice (6-8 weeks old) and incubated with $20 \mathrm{ng} / \mathrm{ml} \mathrm{GM-CSF}$ for 7 days. Then, BMDMs were treated with $100 \mathrm{ng} / \mathrm{ml}$ MTB Hsp16.3, 100 $\mathrm{ng} / \mathrm{ml}$ IFN- $\gamma$, or $100 \mathrm{ng} / \mathrm{ml} \mathrm{IL-4} \mathrm{for} \mathrm{0-72} \mathrm{h.} \mathrm{a} \mathrm{The} \mathrm{morphology} \mathrm{of} \mathrm{BMDMs} \mathrm{incubated} \mathrm{with} \mathrm{IFN-} \gamma$, IL-4, or MTB Hsp16.3 for $12 \mathrm{~h}$. The images were captured under an inverted microscope $(\times 200)$; the picture in the upper right corner is $\times 400$. Total RNA was extracted from the cells using TRIzol according to the manufacturer's instructions. b The mRNA expression levels of iNOS, IL-6, TNF- $\alpha$, Arg-1, IL-10, and TGF- $\beta$ in macrophages by RT-PCR. $\mathbf{c}$ The percentage of F4/80 and NOS2 or CD206 double-positive macrophages was measured by FCM. $\mathbf{d}$ The production of TNF- $\alpha$, IL-10, and TGF- $\beta$ was measured by ELISA. Data are expressed as the mean $\pm \operatorname{SEM}(n=3) . * p<0.05, * * p<0.01, * * * p<0.001$ compared with the control group ( $0 \mathrm{~h}$ group).

binding sites. Then, the membranes were incubated with anti-CCRL2 (Abcam, no. ab88632), anti-CX3CR1 (Abcam, no. ab8021), anti-p38 MAPK (Abcam, no. ab197348), anti-ERK (Abcam, no. 196883), anti-AKT (Abcam, no. ab8805), anti-phospho-ERK (Abcam, no. ab50011), anti-phospho-AKT (Abcam, no. ab81283), anti-phospho-p38-MAPK (Abcam, no. ab47363), and anti-GAPDH (Abcam, no. ab181602) antibodies at $4{ }^{\circ} \mathrm{C}$ overnight according to the manufacturer's instructions. The membranes were then washed with TBST and incubated with a secondary anti-rabbit Ab conjugated to HRP (Cell Signaling Technology, no. 7074s) at room temperature. The signals were detected and analyzed by a chemiluminescence imaging system (ChemiScope5600, CLINX, Shanghai, China), and each experiment was performed in triplicate.

\section{Statistical Analysis}

The data are presented as the mean $\pm \mathrm{SD}$. An unpaired Student's $t$ test was used to evaluate differences between two groups, and a Student's $t$ test was then performed when two conditions were compared, and one-way ANOVA was performed for multiple comparisons followed by a post hoc Tukey's test when necessary. If not, data were analyzed by the Mann-Whitney $U$ test. $p$ values of $<0.05$ were considered significant; two-sided tests were performed. All data analyses were carried out by SPSS 16.0 (SPSS Inc.) and GraphPad Prism ${ }^{\text {TM }} 5.0$ (GraphPad Software Inc.) Statistical software.

\section{RESULTS}

\section{MTB Hsp16.3 Induces Mouse Bone Marrow-Derived Macrophage M2 polarization}

Bone marrow cells were cultured with GM-CSF for 7 days, and CD11b and F4/80 were detected by flow cytometry, showing $90.7 \%$ of cells to be double positive. The results showed that BMDMs were induced successfully (not shown in the figure). To verify the impact of 
b
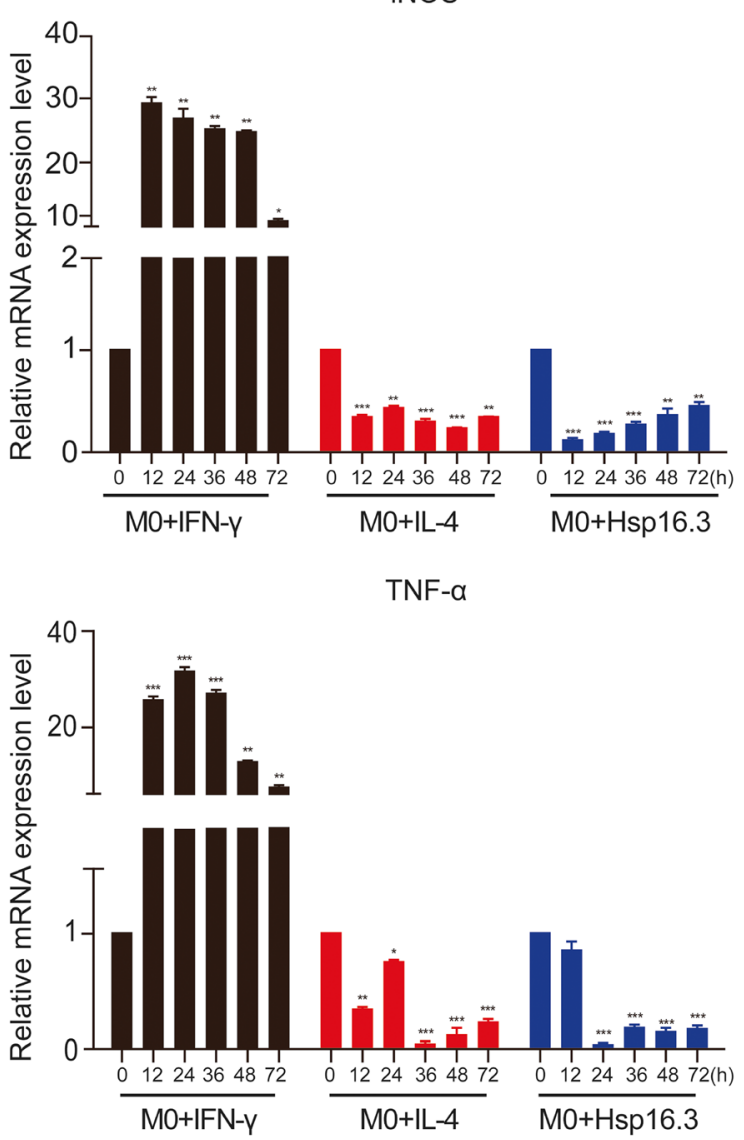

IL-10

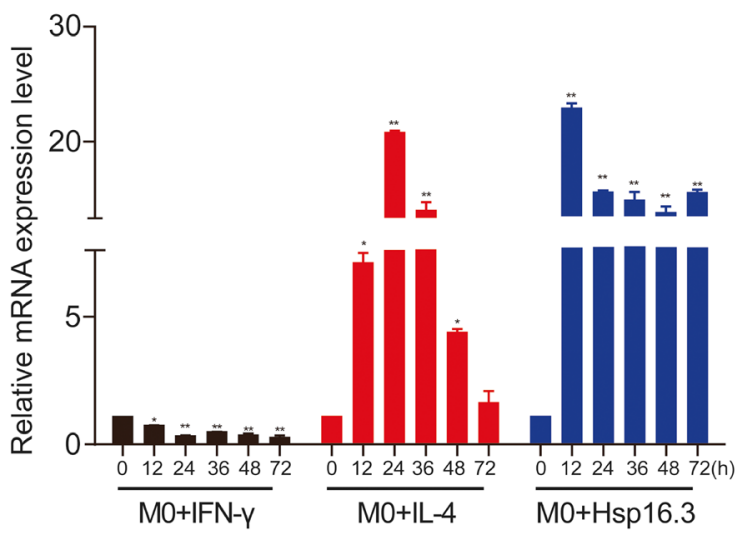

Fig. 1. (continued)

MTB Hsp16.3 on macrophage polarization, BMDMs were incubated with $100 \mathrm{ng} / \mathrm{ml}$ IFN- $\gamma, 10 \mathrm{ng} / \mathrm{ml} \mathrm{IL-4,}$ and $100 \mathrm{ng} / \mathrm{ml}$ MTB Hsp16.3 for $12 \mathrm{~h}, 24 \mathrm{~h}, 36 \mathrm{~h}, 48 \mathrm{~h}$, and $72 \mathrm{~h}$. First, we observed the morphology of macrophages. Compared with the untreated macrophages (Fig.

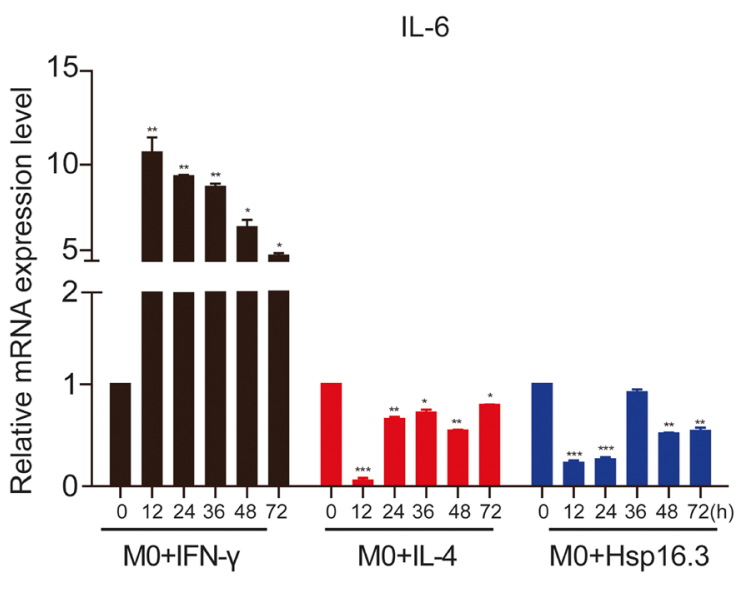

Arg-1

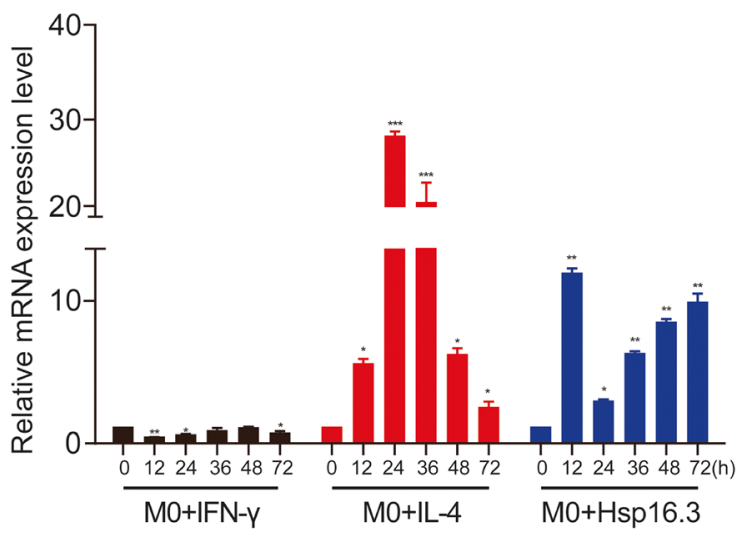

TGF- $\beta$

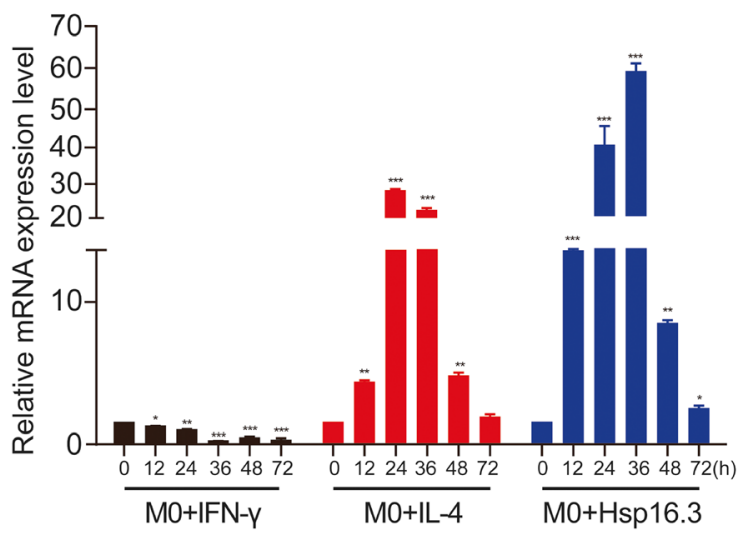

1a), M1 macrophages (incubated with IFN- $\gamma$ ) had an elongated fibroblast-like morphology, whereas BMDMs incubated with MTB Hsp16.3 extended pseudopodia to the same extent as M2 macrophages (incubated with IL4). Therefore, we speculated that MTB Hsp16.3 may 

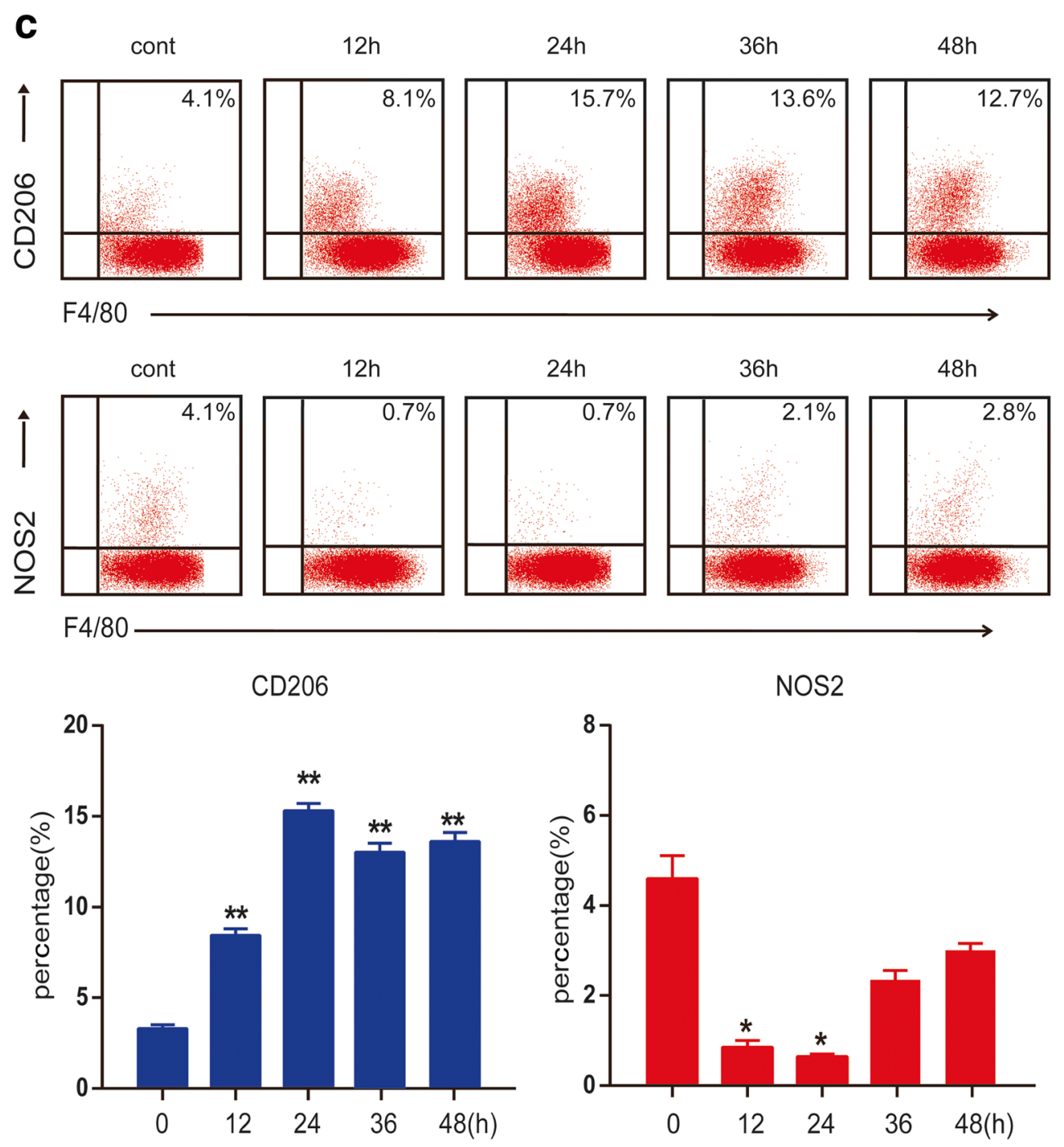

Fig. 1. (continued)

induce the M2 phenotype in macrophages. Studies have reported that macrophages polarized to different phenotypes exhibit a distinct gene expression signature that can be used to identify them [11]. M1 phenotype macrophages secrete the inflammatory cytokines TNF- $\alpha$, IL12 , IL-6, and iNOS. M2 phenotype macrophages produce IL-10 and TGF- $\beta$ and show increased Arg-1 expression. In addition, M2 macrophage polarization can be defined based on a specific genetic signature characterized by the upregulation of Ym1 and Fizz1 expression. To verify which phenotype MTB Hsp16.3 treatment induces, we determined the expression of macrophage phenotype related markers. The mRNA expression levels of Arg-1, TGF- $\beta$, IL-10, iNOS, TNF- $\alpha$, and
IL-6 were determined by real-time PCR. The results showed that the Hsp16.3-treated group had significantly reduced expression levels of iNOS, TNF- $\alpha$, and IL- 6 mRNA (Fig. 1b) and upregulated expression levels of Arg-1, TGF- $\beta$, and IL-10 mRNA (Fig. 1b), as did the IL4-treated BMDMs (M2 macrophages). We also found that the expression levels of Arg-1 and IL-10 peaked at $12 \mathrm{~h}$, and TNF- $\alpha$ expression peaked at $24 \mathrm{~h}$. Compared with those in the untreated group, the expression levels of iNOS and IL- 6 in the treated group were suppressed at $12 \mathrm{~h}$, and TGF- $\beta$ expression was suppressed at $36 \mathrm{~h}$. To investigate the macrophage surface molecules, flow cytometry was used to measure the expression levels of CD206 and NOS2. The +MTB Hsp16.3 group exhibited 

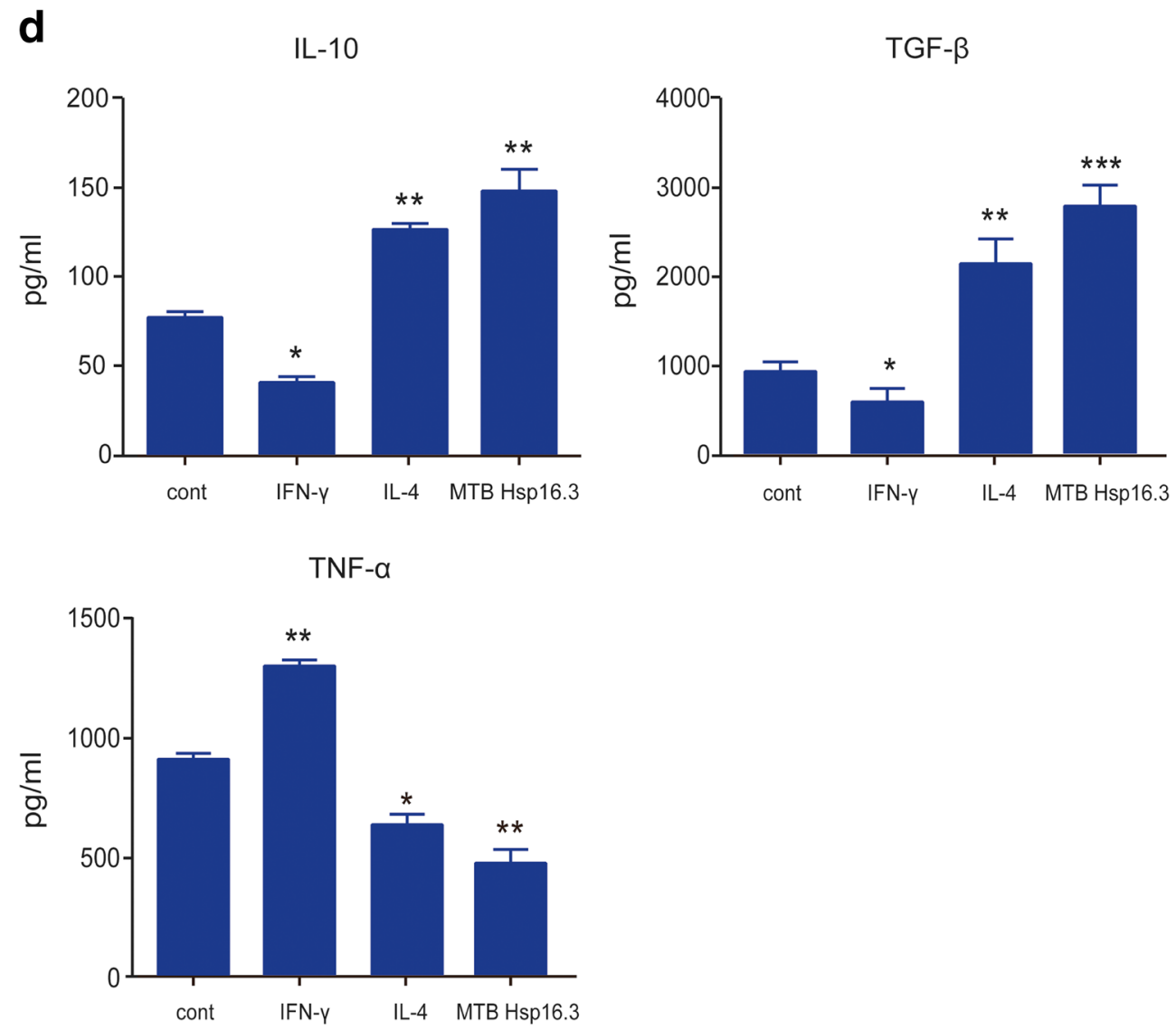

Fig. 1. (continued)

a markedly attenuated percentage of F4/80 and NOS2 double-positive cells (Fig. 1c). The percentage of F4/80 and CD206 macrophages was significantly increased in the MTB Hsp16.3 group compared with the untreated group (Fig. 1c). Then, we assessed if the M2-like cytokines had the same changes. ELISAs were performed to determine the expression of TNF- $\alpha$, IL-10, and TGF- $\beta$ (Fig. 1d). The results were as we expected, compared with untreated group, because the +MTB Hsp16.3 group showed increased production of IL-10 and TGF- $\beta$ and reduced secretion of TNF- $\alpha$. These results indicate that macrophages incubated with MTB Hsp 16.3 recombinant protein show markedly upregulated expression of M2 phenotype markers.

\section{Genome-wide Microarray Analysis of BMDMs Incubated with MTB Hsp16.3}

To explore which receptor is affected by Hsp 16.3 to promote BMDM M2 polarization, a genome-wide microarray was performed to investigate the variation in gene expression between the untreated group and the + Hsp16.3 group. The results showed that the gene expression of the two groups was relatively discrete (Fig. 2a, b). The number of genes with twofold upregulated expression was 568, and the number with twofold downregulated expression was 534 (Fig. 2c). To further elucidate the potential molecular mechanism by which Hsp16.3 induces macrophage M2 phenotype polarization, we examined the related literature and identified 5 genes, including CCRL2, CCR2, CX3CR1, AhR, and Mertk [12-16]. The results revealed that the expression of these genes changed approximately twofold between the two groups (Fig. $2 \mathrm{~d})$. We verified the expression of these 5 predicted target genes. We found that the CX3CR1 and CCRL2 mRNA expression levels were significantly upregulated in the + Hsp 16.3 and more than 5 times greater than those the untreated group from 12 to $48 \mathrm{~h}$ (Fig. 2e). We 
a

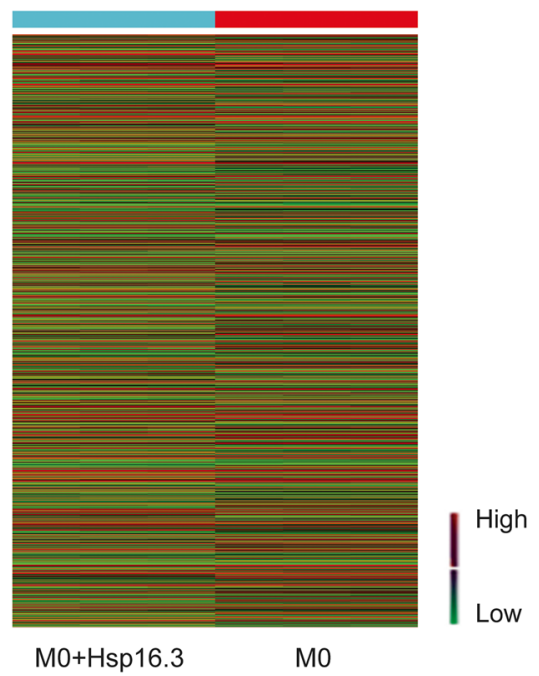

C

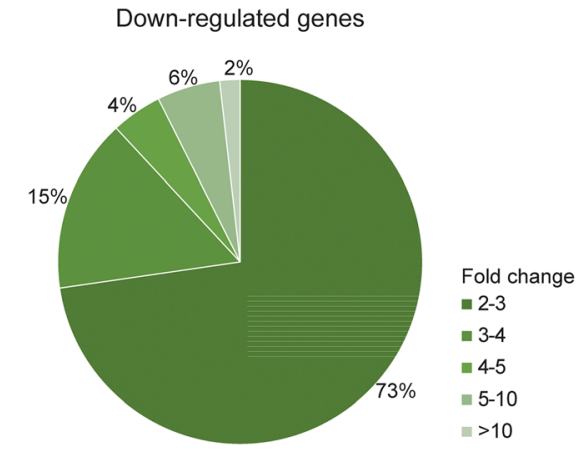

b

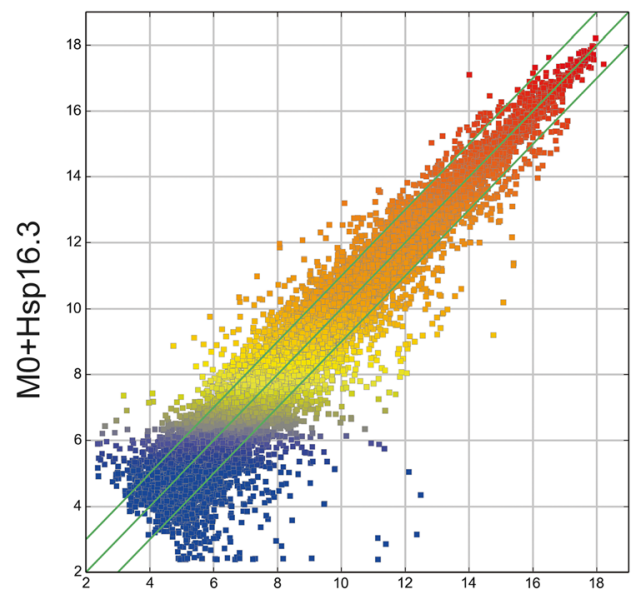

M0

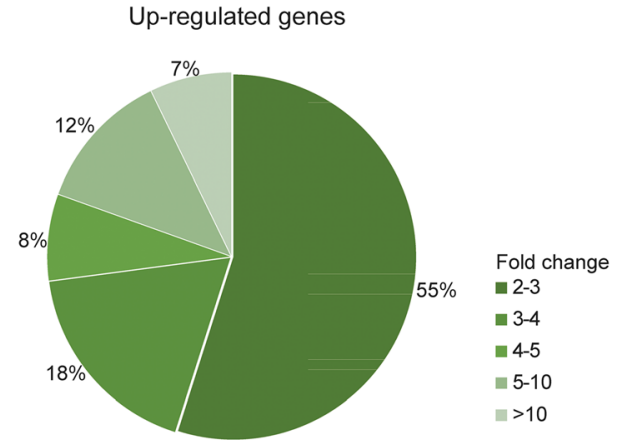

d

\begin{tabular}{|l|l|l|}
\hline Seq-ID & Gene name & $\begin{array}{l}\text { Absolute Fold Change of } \\
\text { M0/and M0+MTB Hsp16.3 }\end{array}$ \\
\hline 11622 & AHR & 4.2 \\
\hline 12772 & CCR2 & 2.034 \\
\hline 54199 & CCRL & 2.668 \\
\hline 13051 & CX3CR1 & 5.489 \\
\hline 17289 & Mertk & 2.54 \\
\hline
\end{tabular}

Fig. 2. Genome-wide microarray analysis of BMDMs incubated with MTB Hsp16.3. Bone marrow cells were isolated from the tibias and femurs of BALB/c mice (6-8 weeks old) and incubated with $20 \mathrm{ng} / \mathrm{ml} \mathrm{GM-CSF}$ for 7 days. Then, BMDMs were treated with $100 \mathrm{ng} / \mathrm{ml} \mathrm{MTB} \mathrm{Hsp} 16.3$ for $72 \mathrm{~h}$, and the cells were collected. Global gene expression was analyzed by a cDNA chip array. a Heat map. b Scatterplot of gene expression. $\mathbf{c}$ The fold change and frequency. d Prediction of 5 target genes, including AHR, CCR2, CCRL2, CX3CR1, and Mertk. e BMDMs were treated with 100 ng/ml MTB Hsp16.3 for 72 h, cells were collected, and the mRNA expression of the indicated genes was determined by real-time PCR. fCells were harvested in RIPA buffer containing protease and phosphatase inhibitor cocktails, and the protein expression levels of CCRL2 and CX3CR1 were determined by Western blot. Data are presented as the mean $\pm \operatorname{SEM}(n=3) . * p<0.05, * * p<0.01, * * * p<0.001$ compared with the control group $(0 \mathrm{~h}$ group). 

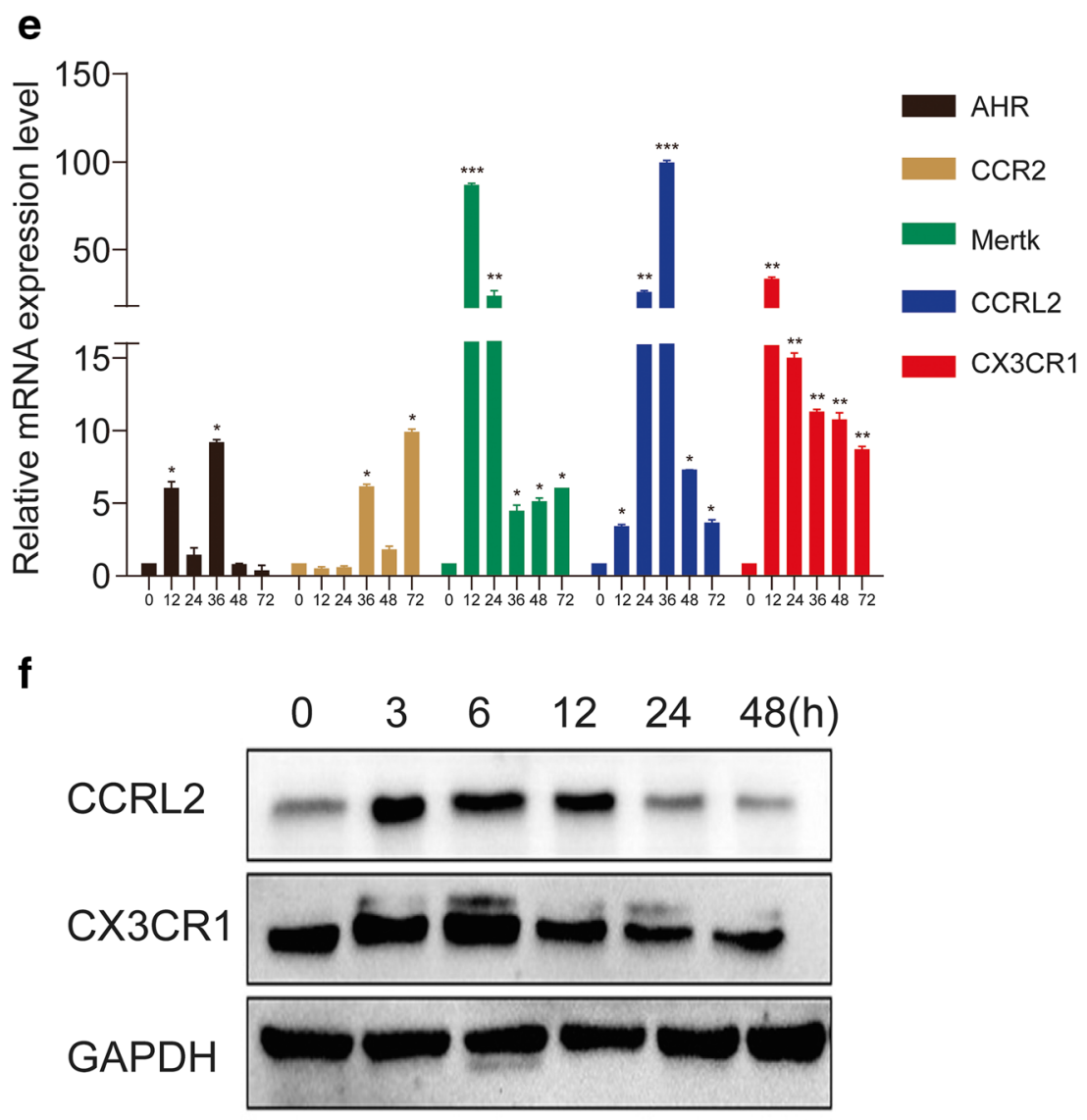

Fig. 2. (continued)

also investigated the production of CX3CR 1 and CCRL2, which peaked from 3 to $6 \mathrm{~h}$ in the treated group compared with the untreated group (Fig 2f). Collectively, our data indicate that Hsp16.3 might upregulate the expression of CX3CR1 and CCRL2 to polarize the macrophage M2 phenotype.

\section{Silencing of CCRL2/CX3CR1 Abrogates the MTB Hsp16.3-Induced Polarization of Macrophages to the M2 Phenotype}

To further confirm whether CCRL2 and CX3CR1 contribute to the MTB Hsp16.3-induced polarization of macrophages to the M2 phenotype, we constructed sequences to silence CCRL2 and CX3CR1. We found that in the targeting of CCRL2, the silencing efficiency of the second sequence pair was the greatest, whereas the first sequence pair had the greatest CX3CR1 silencing effect (Fig. 3a). The production of CCRL2 and CX3CR1 was significantly suppressed after silencing (Fig. 3b). These results indicate that we silenced CCRL2 and CX3CR1 successfully. Then, we determined the changes in the expression levels of M2 phenotype-related cytokines. The results showed that in the knockdown group, the mRNA expression levels of Arg-1, TGF- $\beta$, IL-10, and Ym-1 were inhibited compared to those in the control group (Fig. 3c). NOS2 expression increased, and CD206 expression decreased (Fig. 3d). Compared with the untreated BMDMs, BMDMs transfected with siRNA targeting CCRL2 or CX3CR1 showed significantly decreased production of IL-10 and TGF- $\beta$ (Fig. 3e) and increased TNF- $\alpha$ production (Fig. 3e) after incubation with MTB Hsp16.3. These results demonstrate that MTB Hsp16.3 may induce BMDM polarization to the M2 phenotype through CCRL2 and CX3CR1.

An increasing body of literature has documented that macrophage polarization is regulated through various signaling pathways, such as the AKT and ERK 


\section{a}

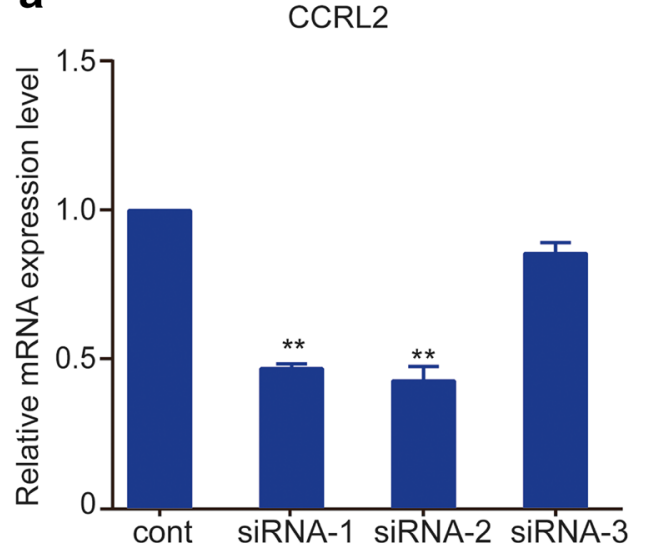

CX3CR1

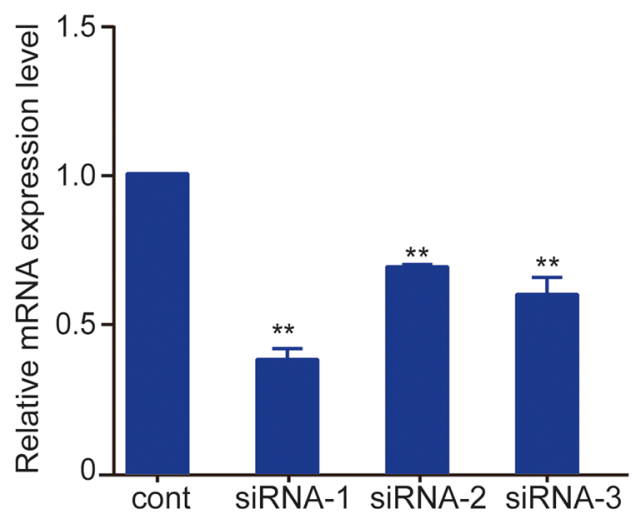

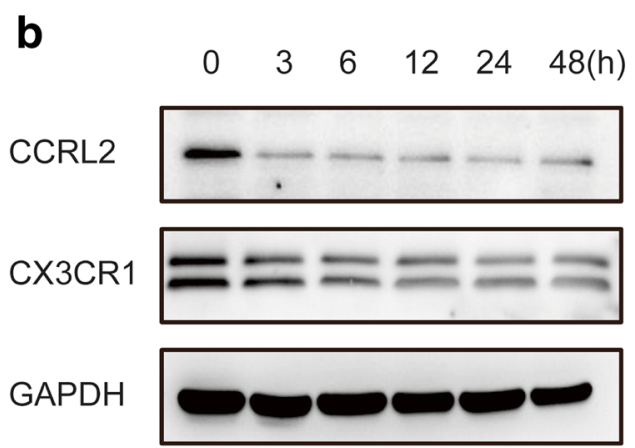

Fig. 3. The silencing of CCRL2/CX3CR1 abrogates the MTB Hsp16.3-induced polarization of macrophages to the M2 phenotype. Bone marrow cells were isolated from the tibias and femurs of BALB/c mice (6-8 weeks old) and incubated with $20 \mathrm{ng} / \mathrm{ml} \mathrm{GM-CSF}$ for 7 days. Then, BMDMs were transfected with siRNA targeting CCRL2 and CX3CR1. a mRNA expression levels of CCRL2 and CX3CR1 were measured by real-time PCR after transfection. b Western blot analysis of CCRL2 and CX3CR1 in the untreated BMDM group and +Hsp16.3 BMDM group transfected with siRNA targeting mouse CCRL2 and CX3CR1 for 0-48 h. $\mathbf{c}$ Real-time PCR analysis of mRNA expression levels of Arg-1, IL-10, TGF- $\beta$, and YM-1 in untreated BMDMs and Hsp16.3-treated BMDMs transfected with siRNA targeting mouse CCRL2 and CX3CR1 for 0-72 h. d FCM analysis of the expression levels of NOS2 and CD206 in the untreated BMDM group and + Hsp16.3 BMDM group transfected with siRNA targeting mouse CCRL2 and CX3CR1 for 0-48 h. e ELISA analysis of the secretion levels of IL-10, TGF- $\beta$, and TNF- $\alpha$ in the untreated BMDM group and +Hsp16.3 BMDM group transfected with siRNA targeting mouse CCRL2 or CX3CR1 for $48 \mathrm{~h}$. f Western blot analysis of p-ERK, ERK, p-AKT, AKT, p-p-38 MAPK, p-38 MAPK in the +Hsp16.3 BMDM group, and +Hsp16.3 BMDM group transfected with siRNA targeting mouse CCRL2 or CX3CR1 at different time points. Data are presented as the mean $\pm \operatorname{SEM}(n=3) . * p<0.05$, $* * p<0.01, * * * p<0.001$ compared with the control group ( $0 \mathrm{~h}$ group).

pathways [17-22]. Thus, to further investigate the downstream signaling in MTB Hsp16.3-induced M2like macrophages, we analyzed the expression of phosphorylated AKT, phosphorylated ERK, and phosphorylated $\mathrm{p} 38$-MAPK in the untreated BMDM group and the +Hsp16.3 BMDM group transfected with siRNA targeting CCRL2 or CX3CR1. The data showed that the levels of p-ERK and p-p38-MAPK were significantly increased in the +Hsp16.3 group (Fig. 3f), whereas the levels were significantly decreased in the + Hsp16.3 BMDM group transfected with siRNA targeting CCRL2; the production of phosphorylated
ERK, phosphorylated p38-MAPK, and phosphorylated AKT in the +Hsp16.3 BMDMs transfected with siRNA targeting CX3CR1 was significantly decreased. These results suggest that MTB Hsp16.3 polarizes BMDMs to the M2 phenotype in vitro by altering the signal transduction of the AKT/ERK/p38-MAPK pathway.

\section{CCRL2/CX3CR1 Overexpression Promotes the Macrophage M2 Phenotype}

To verify the effect of CCRL2 and CX3CR1 on Hsp16.3-induced polarization of macrophages to the 
e

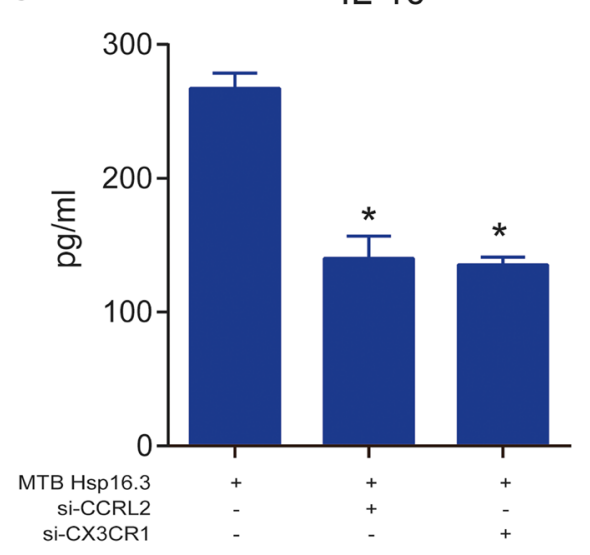

TNF- $\alpha$

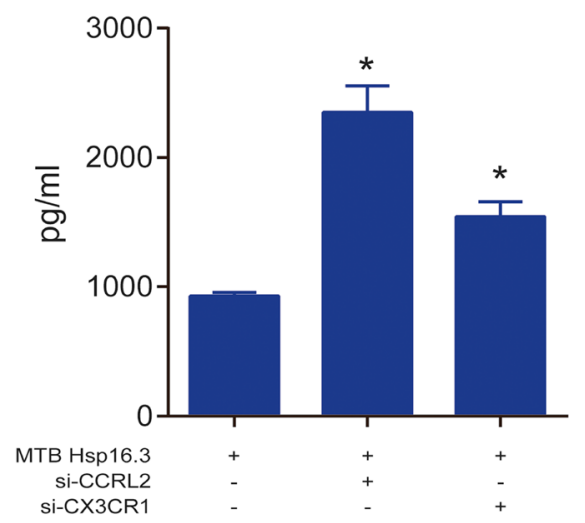

TGF- $\beta$

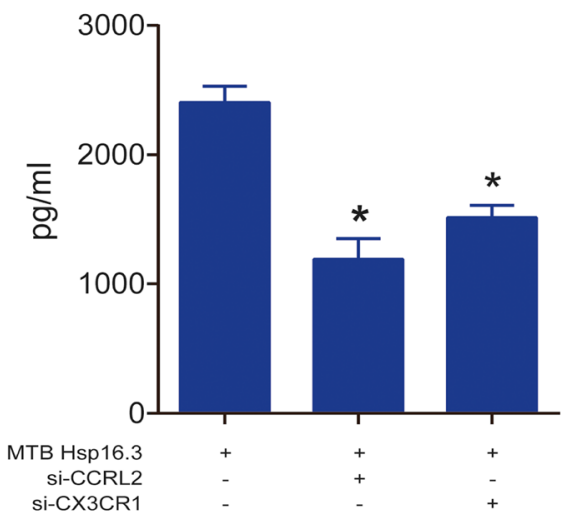

$\mathbf{f}$

f
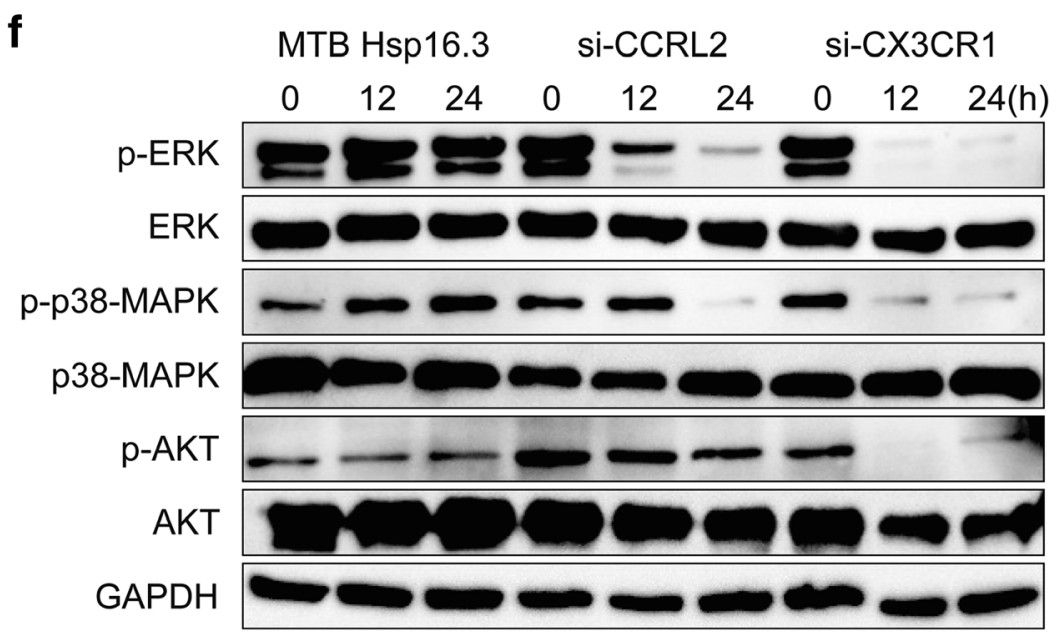

Fig. 3. (continued)

M2 phenotype, we constructed pcDNA3.1-CCRL2 (termed p-CCRL2) and pcDNA3.1-CX3CR1 (termed p-CX3CR1) overexpression vectors (Fig. 4a). Two colonies were selected from the positive colonies and cultured in LB medium (+Amp) for 8-12 $\mathrm{h}$, and then the plasmids were extracted. The extracted 
C
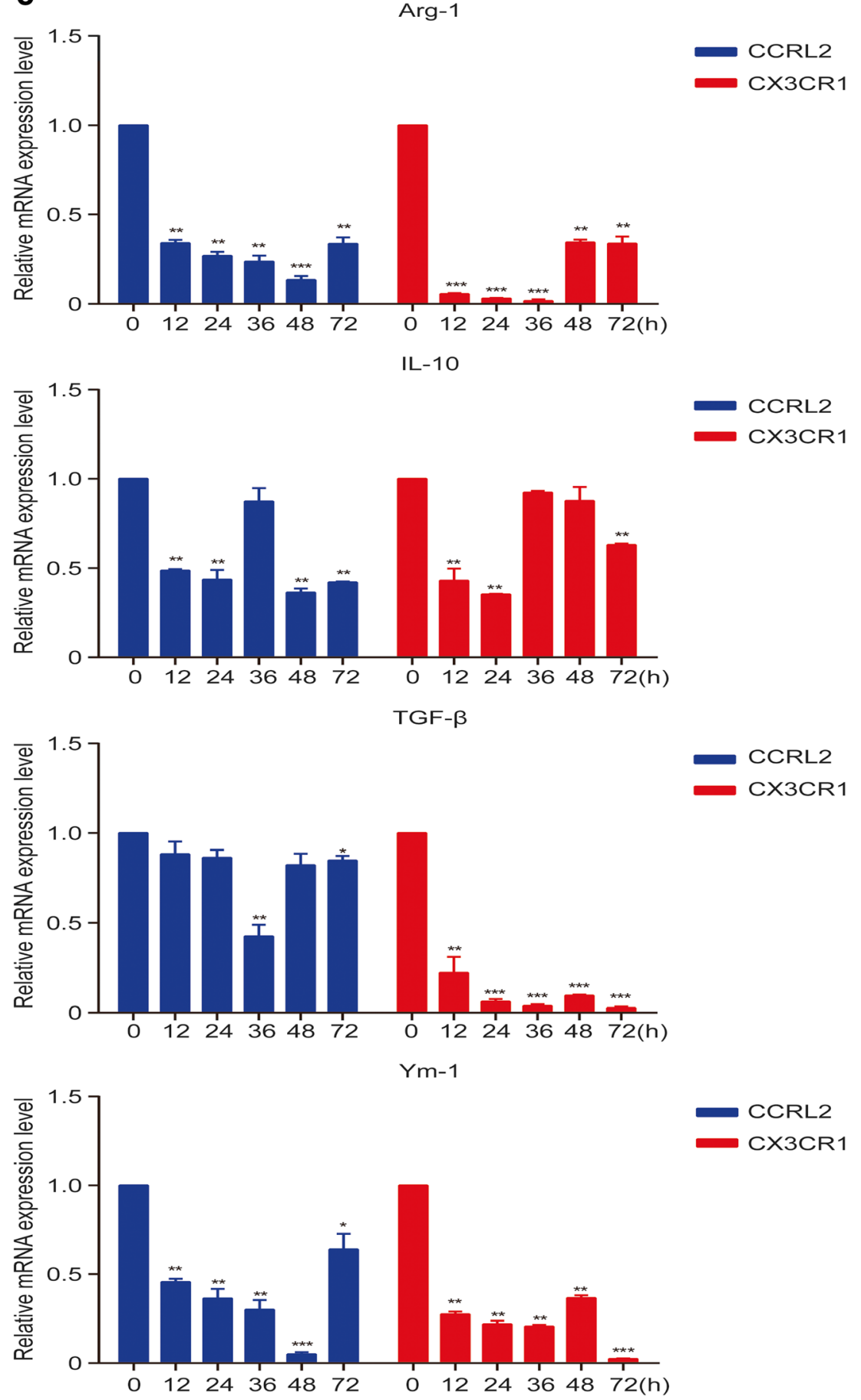

Fig. 3. (continued)

recombinant plasmids were identified by BamHI and HindIII digestion (Fig. 4b). The results showed that the target bands appeared at $5427 \mathrm{bp}, 1065 \mathrm{bp}$, and
$1083 \mathrm{bp}$ after double digestion. The positive plasmid was verified by double restriction enzyme digestion, and the DNA sequence was sent to Hua Da Inc. for 


\section{d}

cont

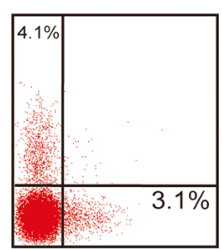

cont

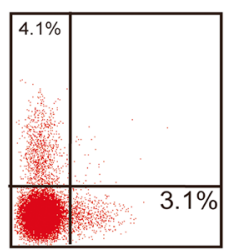

cont

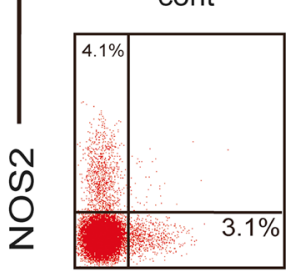

$12 \mathrm{~h}$

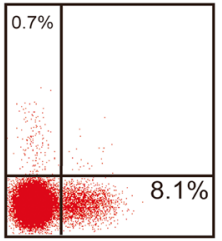

Si-CX3CR1-12h
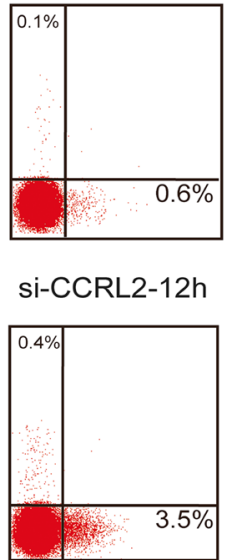

$24 \mathrm{~h}$

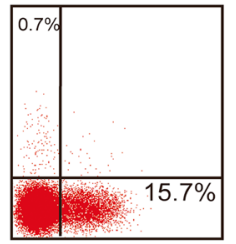

si-CX3CR1-24h

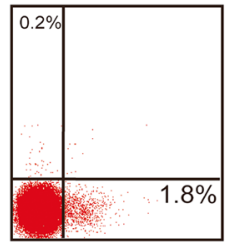

si-CCRL2-24h

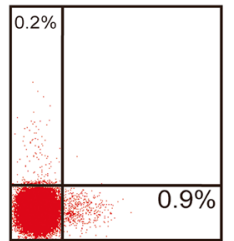

$36 \mathrm{~h}$
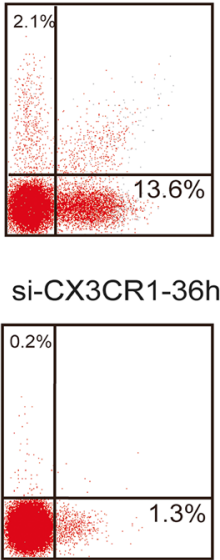

Si-CCRL2-36h

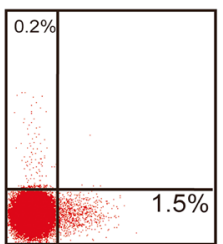

$48 \mathrm{~h}$

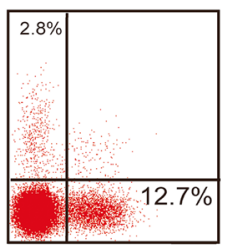

si-CX3CR1-48h

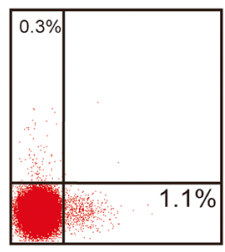

si-CCRL2-48h

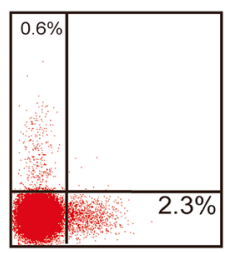

\section{CD206}
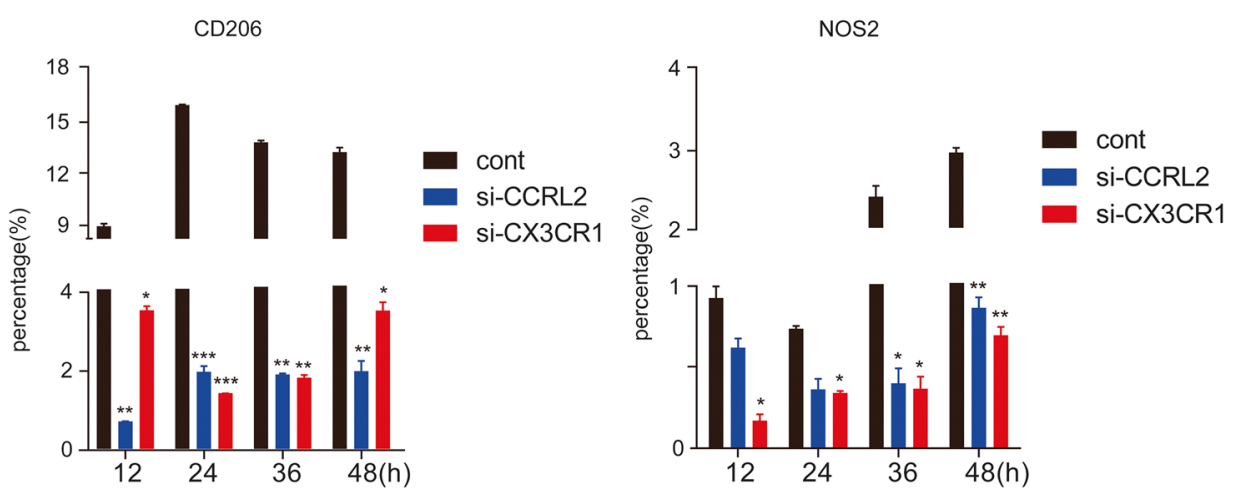

Fig. 3. (continued)

complete sequencing (Fig. 4c). The CCRL2 and CX3CR1 sequences in the plasmid were identical to those in the CDS region sequences of CCRL2 and CX3CR1 in the NCBI database. The results above indicate that the p-CCRL2 and p-CX3CR1 overexpression vectors were constructed successfully.

We investigated the M2-related markers to further demonstrate the effect of CCRL2 and CX3CR1 on macrophage polarization. BMDMs were transiently transfected with p-CCRL2, p-CX3CR1, or p-cont in vitro for $48 \mathrm{~h}$ and then incubated with $100 \mathrm{ng} / \mathrm{ml}$ MTB Hsp16.3 for $0-72 \mathrm{~h}$. The results showed that the mRNA expression levels of Arg-1, IL-10, and TGF- $\beta$ mRNA in the plasmid-transfected groups were significantly upregulated compared with those in the +Hsp16.3 group (Fig. 4d). Compared with the +Hsp16.3 control macrophages, macrophages transiently transfected with p-CCRL2 or p-CX3CR1 incubated with Hsp16.3 showed increased production of CD206 (Fig. 4e), IL-10, and TGF- $\beta$ (Fig. 4f), and 
a

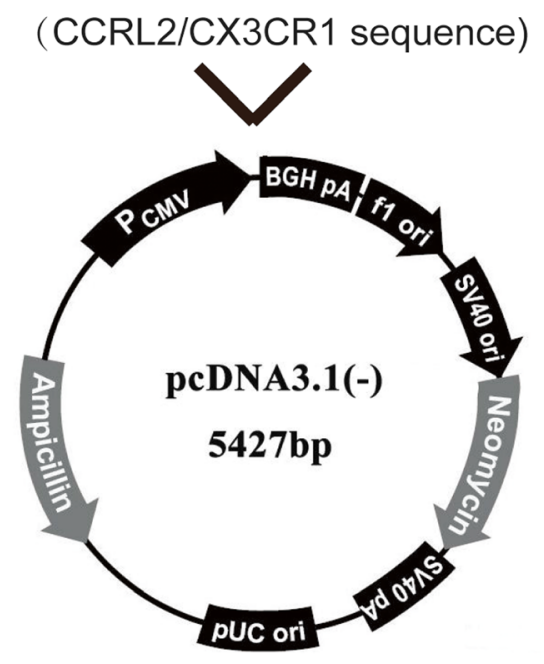

b
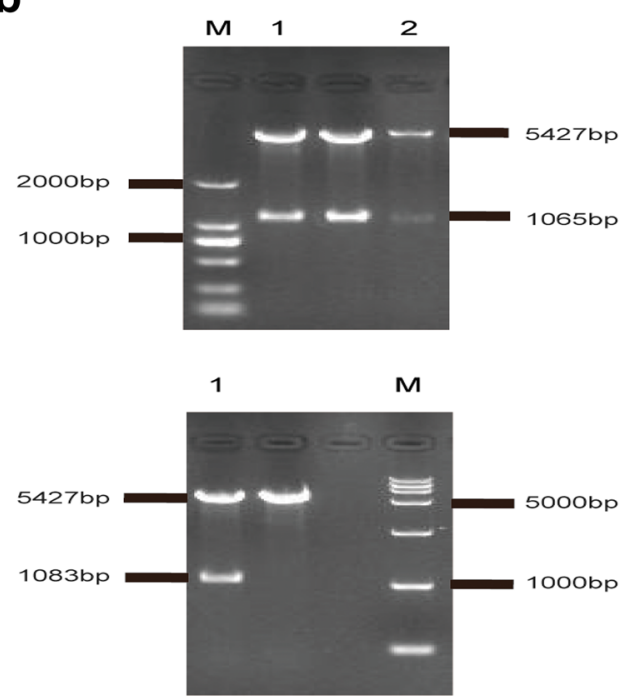

C

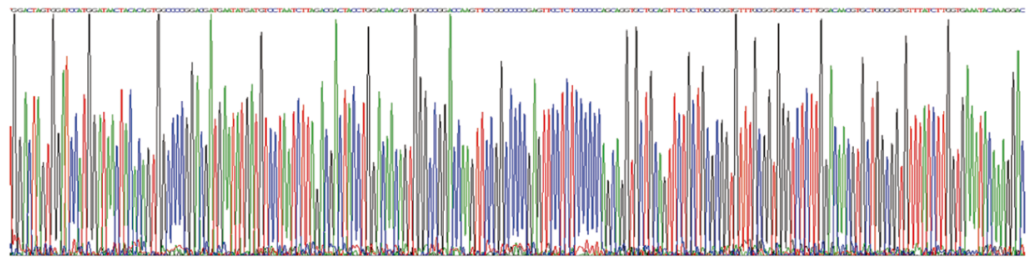

Query 1091 ATGGATAACTACACAGTGGCCCCGGACGATGAA 1124

Sbjct 2140 TGAACTGTACAGCAATTTGCATCAAAGGCAGGA 2173

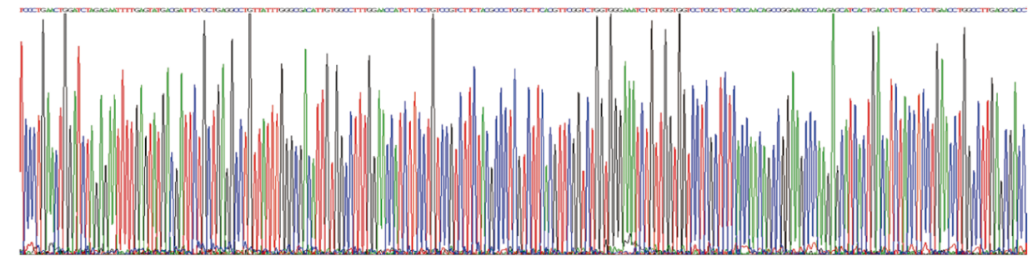

Query 15961 ATGTCCACCTCCTTCCCTGAACTGGATCTAGAG 15994

Sbjct 16981 GTTTCACTCACTACACAAGCGAGGGAGATGGG 17014

Fig. 4. CCRL2/CX3CR1 overexpression promotes the macrophage M2 phenotype. a The schematic structure of pcDNA3.1-CCRL2 (termed p-CCRL2) and pcDNA3.1-CX3CR1 (termed p-CX3CR1). b Identification of pcDNA3.1-CCRL2 and pcDNA3.1-CX3CR1 by BamHI and HindIII double enzyme digestion. $\mathbf{c}$ DNA sequence of the recombinant plasmid. BMDMs were transiently transfected with p-CCRL2, p-CX3CR1, or p-cont in vitro and then incubated with $100 \mathrm{ng} / \mathrm{ml}$ MTB Hsp16.3 for 0-48 h. d The mRNA expression levels of IL-10, Arg-1, and TGF- $\beta$ were determined by real-time PCR. e The production of NOS2 or CD206 in F4/80-positive macrophages was measured by FCM. $\mathbf{f}$ The secretion levels of IL-10, TGF- $\beta$, and TNF- $\alpha$ were measured by ELISA at $48 \mathrm{~h}$. The data are presented as the mean $\pm \operatorname{SEM}(n=3) . * p<0.05, * * p<0.01, * * * p<0.001$ compared with the control group $(0 \mathrm{~h}$ group).

significantly decreased production of TNF- $\alpha$. Taken together, these results show that MTB Hsp16.3 induces macrophage M2 phenotype polarization through CCRL2 and CX3CR1. 

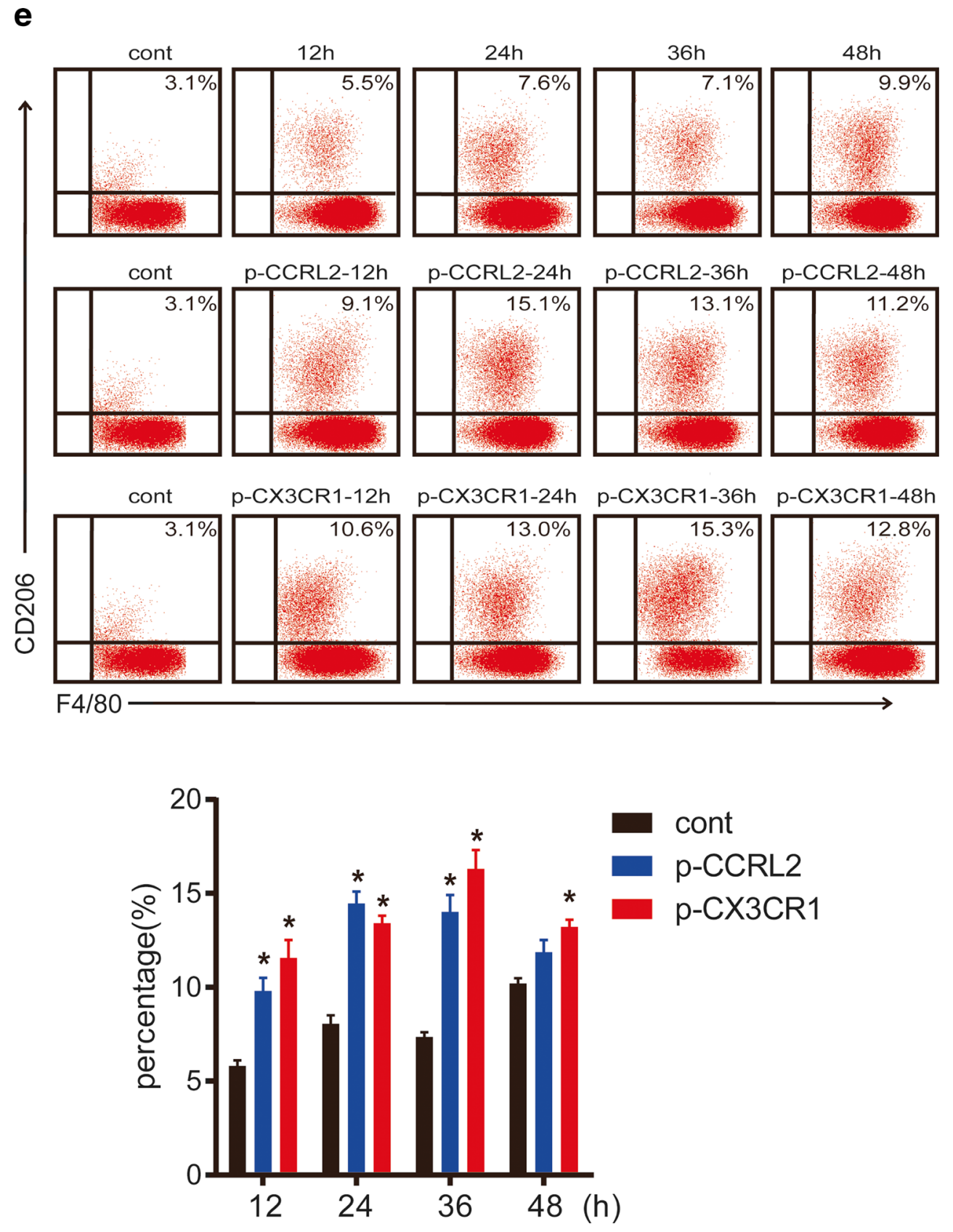

Fig. 4. (continued)

\section{DISCUSSION}

It is well known macrophages activated by danger signals of their own or pathogen sources can be polarized into different subtypes [23]. According to their function, macrophages can be divided into M1 or M2 types. Ml macrophages have enhanced phagocytosis, inflammatory cytokine secretion, and antigen presentation abilities [24]. M2 macrophages can promote wound repair and fibrosis, mediate the escape of tumors and pathogens, and participate in Th2-type immune responses [25]. In long-term latent MTB infections, macrophages lose their ability to present antigens; therefore, $\mathrm{T}$ cells do not acquire effector signals 

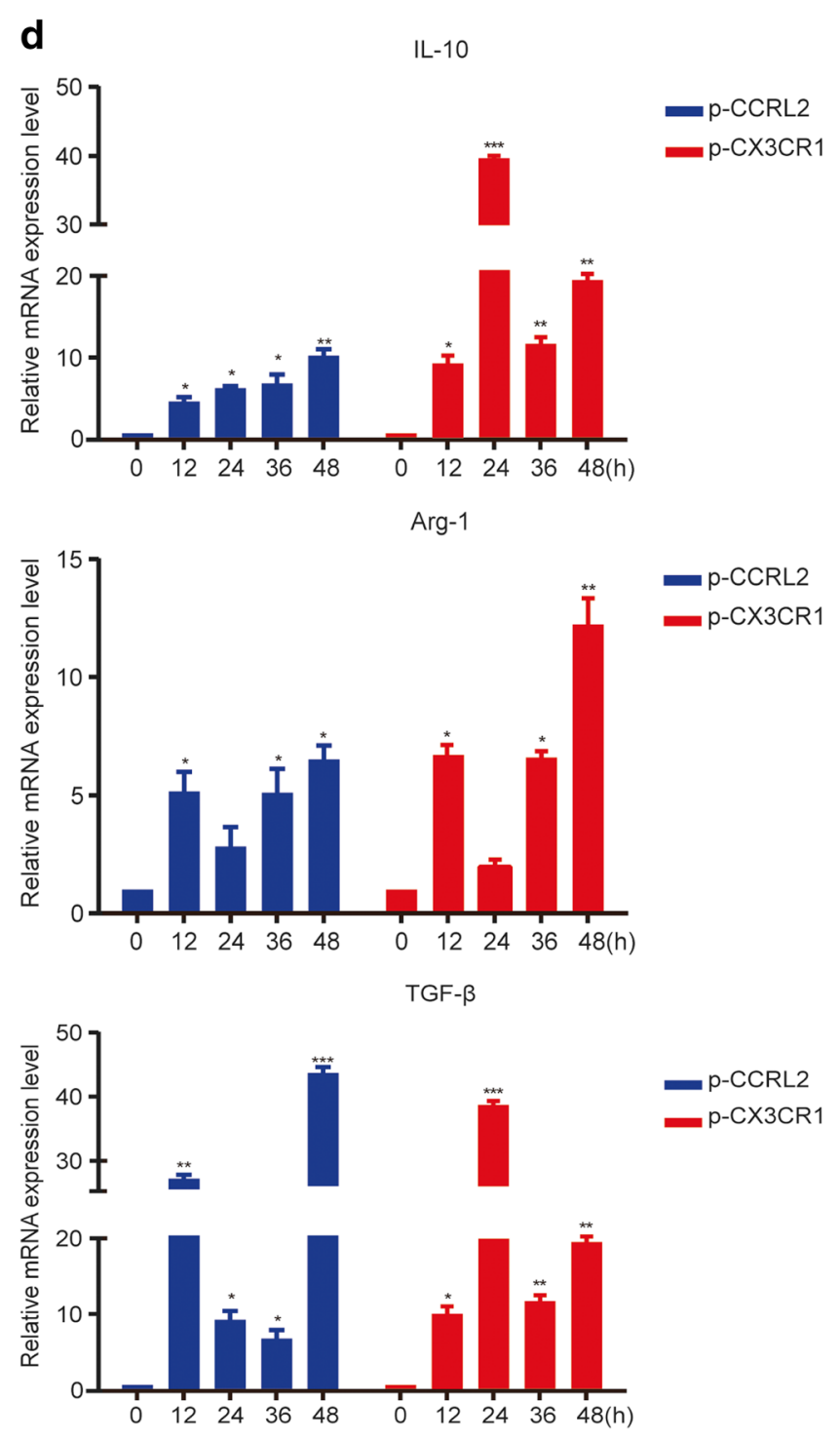

Fig. 4. (continued)

to secrete IFN- $\gamma$ and macrophages are not stimulated. In this microenvironment, macrophages polarized to the M2 phenotype are inhabited by MTB. And many studies have shown that MTB Hsp16.3 expression is increased in the latent infection phase [26], so we surmise that MTB Hsp16.3 might induce macrophage M2 polarization. There were reports about MTB Hsp16.3 inhibiting autophagy and apoptosis [27] but few reports on macrophage polarization. In a previous study, we have expressed and purified MTB Hsp16.3 protein in vitro [10]. Based on these findings, in this study, we explored the impact of MTB Hsp16.3 on macrophage polarization.
Our results revealed that MTB Hsp16.3 treatment upregulates the production of M2-related markers in mouse bone marrow-derived macrophages. The secretion of inflammatory cytokines IL- 6, TNF- $\alpha$, and iNOS forms an inflammatory microenvironment that promotes the polarization of macrophages to the M1 phenotype, forming a proinflammatory positive feedback loop. The production of IL-10, TGF- $\beta$, and Arg-1 further promotes the polarization of macrophages to the M2 type [28], also forming a positive feedback mechanism for the anti-inflammatory response. Our results revealed that IL-6, TNF-a, iNOS, IL-10, TGF- $\beta$, and Arg-1 mRNA expression levels were dynamically 
f

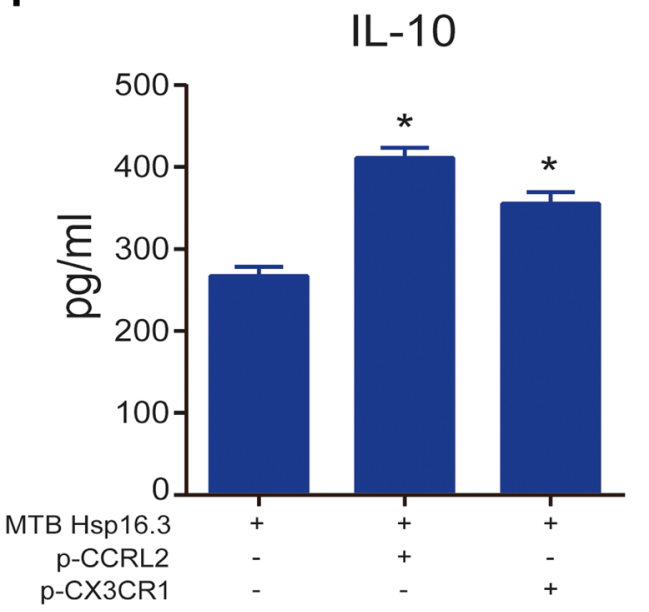

TNF- $\alpha$

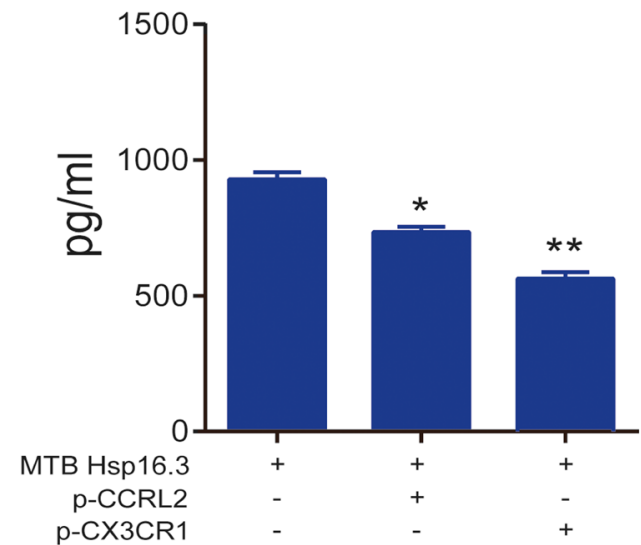

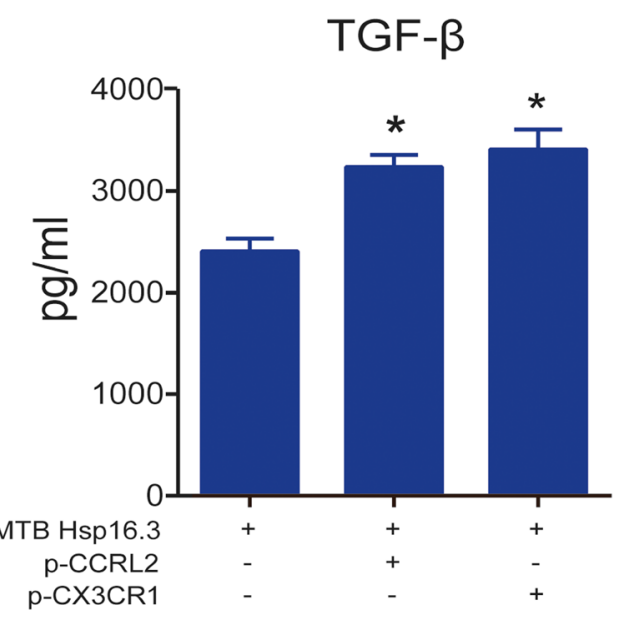

p-CX3CR1 
"atypical chemokine receptor" (ACKR), which lacks classical GPCR signaling and chemotactic activity and has been shown to limit inflammation through its ability to clear chemokines in areas of inflammation. In addition, CCRL2 is critical for the development of Th2 responses [34], and it has been reported that CCRL2 is associated with macrophage M2 polarization [35]. CX3CR1, the receptor of fractalkine, is also an important chemokine receptor for macrophages. CX3CR1 mediates monocyte patency in the vascular space under steady-state condition, recruits tissue-resident macrophages [29], and regulates tissue macrophage function during various disease processes. Among the innate immune cells, macrophages expressing the chemokine receptor CX3CR1 contribute to maintaining the inflammatory response balance in the gut by producing the anti-inflammatory cytokine IL-10 [36]. In addition, CX3CR1 ablation inhibits the macrophage-mediated repair of acute skeletal muscle damage [37]. Therefore, CX3CR1 may be involved in macrophage M2 polarization. However, there is also evidence that CX3CR1 promotes macrophage M1 polarization [38, 39]. Therefore, the effect of CX3CR1 on macrophage polarization is not clear. In our work, we used a cDNA microarray to analyze the differential expression of genes between the $+\mathrm{Hsp} 16.3$ group and the untreated group, and we consulted the relevant literature to identify CCRL2 and CX3CR1, which may be involved in macrophage polarization. We found that MTB Hsp16.3 treatment increased the expression of CCRL2 and CX3CR1 at 3-6 h. Overexpression and silencing techniques further validated that MTB Hsp16.3 induces macrophage M2 polarization via CCRL2 and CX3CR1. In addition, we explored downstream signaling molecules affected by CCRL 2 and CX3CR1. Because a large number of studies have shown that the AKT/ERK/p38-MAPK signaling pathway plays an important role in the expression of inflammatory factors [40, 41], phagocytosis [42], and resistance to pathogenic bacteria [43, 44], we investigated these signaling molecules. After CCRL2 silencing, p-ERK and p-p38 levels were significantly reduced, and there was no clear change in p-AKT levels. After CX3CR1 silencing, the production of p-ERK, p-p38-MAPK, and pAKT was significantly reduced. These findings suggest that the MTB Hsp16.3-induced M2 polarization of mouse bone marrow-derived macrophages occurs via AKT/ERK/p38-MAPK. In future work, we will provide sufficient proof to indicate how MTB Hsp16.3 interacts with CCRL2/CX3CR1 to induce macrophage M2 polarization, and the downstream signaling will be further verified by blocking the AKT/ ERK/p38-MAPK signaling pathway.

In conclusion, we found that MTB Hsp16.3 promotes the polarization of BMDMs to the M2 phenotype via CCRL2/CX3CR1 and may be mediated by the AKT/ ERK/p38-MAPK signaling pathway. Our results might explore the potential role of Hsp16.3 in LTBI. And we believe an in-depth understanding of the relationship between the polarization of macrophages and MTB interactions will help to improve tuberculosis prevention and control.

\section{FUNDING INFORMATION}

This work was supported by the National Nature Science Foundation of China (no. 81860291) and the Science and Technology project of Guizhou (no. [2018]1192).

Open Access This article is distributed under the terms of the Creative Commons Attribution 4.0 International License (http://creativecommons.org/licenses/by/ 4.0/), which permits unrestricted use, distribution, and reproduction in any medium, provided you give appropriate credit to the original author(s) and the source, provide a link to the Creative Commons license, and indicate if changes were made.

\section{REFERENCES}

1. WHO. 2018. Global tuberculosis report. https://www.who.int/tb/ publications/global_rep-ort/en/.

2. Huang, Z., Q. Luo, Y. Guo, J. Chen, G. Xiong, Y. Peng, J. Ye, and J. Li. 2015. Mycobacterium tuberculosis-induced polarization of human macrophage orchestrates the formation and development of tuberculous granulomas in vitro. PLoS One 10 (6): e0129744.

3. Matucci, A., E. Maggi, and A. Vultaggio. 2014. Cellular and humoral immune responses during tuberculosis infection: useful knowledge in the era of biological agents. The Journal of Rheumatology. Supplement 91: 17-23.

4. Kroner, A., A.D. Greenhalgh, J.G. Zarruk, R. Passos Dos Santos, M. Gaestel, and S. David. 2014. TNF and increased intracellular iron alter macrophage polarization to a detrimental M1 phenotype in the injured spinal cord. Neuron 83 (5): 1098-1116.

5. Lawrence, T., and G. Natoli. 2011. Transcriptional regulation of macrophage polarization: enabling diversity with identity. Nature Reviews. Immunology 11 (11): 750-761. 
6. Tomioka, H., Y. Tatano, W.W. Maw, C. Sano, Y. Kanehiro, and T. Shimizu. 2012. Characteristics of suppressor macrophages induced by mycobacterial and protozoal infections in relation to alternatively activated M2 macrophages. Clinical \& Developmental Immunology 2012: 635451

7. Butler, M.G., S.K. Rafi, and A.M. Manzardo. 2015. High-resolution chromosome ideogram representation of currently recognized genes for autism spectrum disorders. International Journal of Molecular Sciences 16 (3): 6464-6495.

8. Schmall, A., H.M. Al-Tamari, S. Herold, M. Kampschulte, A. Weigert, A. Wietelmann, N. Vipotnik, et al. 2015. Macrophage and cancer cell cross-talk via CCR2 and CX3CR1 is a fundamental mechanism driving lung cancer. American Journal of Respiratory and Critical Care Medicine 191 (4): 437-447.

9. Yao, N., J.T. Dong, F. Xu, X.Z. Tian, F. Wu, L. Zhang, J.D. Wu, and W.J. Zhang. 2012. Correlation study between Hsp16.3 of Mycobacterium tuberculosis and the apoptosis of infected macrophage. Chinese Journal of Pathogen Biology (8).

10. Qin, H., S.Y. Gao, J.B. Yuan, L. Xu, and J.M. Luo. 2014. Prokaryotic expression and function detection of Mycobacterium tuberculosis Hsp16.3. Chinese Journal of Cellular and Molecular Immunology 30 (5): 480-484.

11. Azad, A.K., M.V. Rajaram, and L.S. Schlesinger. 2014. Exploitation of the macrophage mannose receptor (CD206) in infectious disease diagnostics and therapeutics. Journal of Cytology \& Molecular Biology 1 (1).

12. Salvi, V., F. Sozio, S. Sozzani, and A. Del Prete. 2017. Role of atypical chemokine receptors in microglial activation and polarization. Frontiers in Aging Neuroscience 9: 148.

13. Zhou, D., C. Huang, Z. Lin, S. Zhan, L. Kong, C. Fang, and J. Li. 2014. Macrophage polarization and function with emphasis on the evolving roles of coordinated regulation of cellular signaling pathways. Cellular Signalling 26 (2): 192-197.

14. Chalmers, S.A., V. Chitu, M. Ramanujam, and C. Putterman. 2015. Therapeutic targeting of macrophages in lupus nephritis. Discovery Medicine 20 (108): 43-49.

15. Bock, K.W. 2019. Human AHR functions in vascular tissue: proand anti-inflammatory responses of AHR agonists in atherosclerosis. Biochemical Pharmacology 159: 116-120.

16. Myers, K.V., S.R. Amend, and K.J. Pienta. 2019. Targeting Tyro3, Axl and MerTK (TAM receptors): implications for macrophages in the tumor microenvironment. Molecular Cancer 18 (1): 94.

17. Vergadi, E., E. Ieronymaki, K. Lyroni, K. Vaporidi, and C. Tsatsanis. 2017. Akt Signaling pathway in macrophage activation and M1/M2 polarization. Journal of Immunology 198 (3): 1006-1014.

18. Covarrubias, A.J., H.I. Aksoylar, and T. Horng. 2015. Control of macrophage metabolism and activation by mTOR and Akt signaling. Seminars in Immunology 27 (4): 286-296.

19. Shan, M., J. Qin, F. Jin, X. Han, H. Guan, X. Li, J. Zhang, H. Zhang, and Y. Wang. 2017. Autophagy suppresses isoprenaline-induced M2 macrophage polarization via the ROS/ERK and mTOR signaling pathway. Free Radical Biology \& Medicine 110: 432-443.

20. Wang, Y., C.C. Han, D. Cui, Y. Li, Y. Ma, and W. Wei. 2017. Is macrophage polarization important in rheumatoid arthritis? International Immunopharmacology 50: 345-352.

21. Mahon, O.R., S. O'Hanlon, C.C. Cunningham, G.M. McCarthy, C. Hobbs, V. Nicolosi, D.J. Kelly, and A. Dunne. 2018. Orthopaedic implant materials drive M1 macrophage polarization in a spleen tyrosine kinase- and mitogen-activated protein kinase-dependent manner. Acta Biomaterialia 65: 426-435.
22. Islam, S.U., J.H. Lee, A. Shehzad, E.M. Ahn, Y.M. Lee, and Y.S. Lee. 2018. Decursinol angelate inhibits LPS-induced macrophage polarization through modulation of the NFkappaB and MAPK signaling pathways. Molecules 23 (8).

23. Mantovani, A., A. Sica, S. Sozzani, P. Allavena, A. Vecchi, and M. Locati. 2004. The chemokine system in diverse forms of macrophage activation and polarization. Trends in Immunology 25 (12): 677-686.

24. Atri, C., F.Z. Guerfali, and D. Laouini. 2018. Role of human macrophage polarization in inflammation during infectious diseases. International Journal of Molecular Sciences 19 (6).

25. Mueller, C.K., and S. Schultze-Mosgau. 2011. Histomorphometric analysis of the phenotypical differentiation of recruited macrophages following subcutaneous implantation of an allogenous acellular dermal matrix. International Journal of Oral and Maxillofacial Surgery 40 (4): 401-407.

26. Panda, A.K., A. Chakraborty, S.K. Nandi, A. Kaushik, and A. Biswas. 2017. The C-terminal extension of Mycobacterium tuberculosis Hsp16.3 regulates its oligomerization, subunit exchange dynamics and chaperone function. The FEBS Journal 284 (2): 277-300.

27. Shi, C.H., Y. Jiang, Y. Zhao, F.F. Mao, C.Q. Zhang, B. Bai, and H. Zhang. 2011. Effects of Mycobacterium tuberculosis Hsp16.3 protein on the autophagy function of mice macrophages. Chinese Journal of Cellular and Molecular Immunology 27 (12): 13011303.

28. Orecchioni, M., Y. Ghosheh, A.B. Pramod, and K. Ley. 2019. Macrophage polarization: different gene signatures in M1(LPS+) vs. classically and M2(LPS-) vs. alternatively activated macrophages. Frontiers in Immunology 10: 1084.

29. Lasky, C.E., R.M. Olson, and C.R. Brown. 2015. Macrophage polarization during murine Lyme borreliosis. Infection and Immunity 83 (7): 2627-2635.

30. Maho, A., A. Bensimon, G. Vassart, and M. Parmentier. 1999. Mapping of the CCXCR1, CX3CR1, CCBP2 and CCR9 genes to the CCR cluster within the 3p21.3 region of the human genome. Cytogenetics and Cell Genetics 87 (3-4): 265-268.

31. Zlotnik, A., and O. Yoshie. 2000. Chemokines: a new classification system and their role in immunity. Immunity 12: 121-127.

32. Monnier, J., S. Lewen, E. O'Hara, K. Huang, H. Tu, E.C. Butcher, and B.A. Zabel. 2012. Expression, regulation, and function of atypical chemerin receptor CCRL2 on endothelial cells. Journal of Immunology 189 (2): 956-967.

33. Zabel, B.A., S. Nakae, L. Zuniga, J.Y. Kim, T. Ohyama, C. Alt, J. Pan, et al. 2008. Mast cell-expressed orphan receptor CCRL2 binds chemerin and is required for optimal induction of IgE-mediated passive cutaneous anaphylaxis. The Journal of Experimental Medicine 205 (10): 2207-2220.

34. Yoshimura, T., and J.J. Oppenheim. 2011. Chemokine-like receptor 1 (CMKLR1) and chemokine (C-C motif) receptor-like 2 (CCRL2); two multifunctional receptors with unusual properties. Experimental Cell Research 317 (5): 674-684.

35. Mazzon, C., L. Zanotti, L. Wang, A. Del Prete, E. Fontana, V. Salvi, P.L. Poliani, and S. Sozzani. 2016. CCRL2 regulates M1/M2 polarization during EAE recovery phase. Journal of Leukocyte Biology 99 (6): 1027-1033.

36. Marelli, G., C. Belgiovine, A. Mantovani, M. Erreni, and P. Allavena. 2017. Non-redundant role of the chemokine receptor CX3CR1 in the anti-inflammatory function of gut macrophages. Immunobiology 222 (2): 463-472. 
37. Zhao, W., H. Lu, X. Wang, R.M. Ransohoff, and L. Zhou. 2016. CX3CR1 deficiency delays acute skeletal muscle injury repair by impairing macrophage functions. The FASEB Journal 30 (1): 380393.

38. Tang, Z., Y. Gan, Q. Liu, J.X. Yin, Q. Liu, J. Shi, and F.D. Shi. 2014. CX3CR1 deficiency suppresses activation and neurotoxicity of microglia/macrophage in experimental ischemic stroke. Journal of Neuroinflammation 11: 26.

39. Ran, L., Q. Yu, S. Zhang, F. Xiong, J. Cheng, P. Yang, J.F. Xu, et al. 2015. Cx3cr1 deficiency in mice attenuates hepatic granuloma formation during acute schistosomiasis by enhancing the M2-type polarization of macrophages. Disease Models \& Mechanisms 8 (7): 691-700.

40. Zhang, D., L. Ren, M. Zhao, C. Yang, X. Liu, H. Zhang, Y. Jiang, et al. 2019. Role of Tim-3 in decidual macrophage functional polarization during abnormal pregnancy with Toxoplasma gondii infection. Frontiers in Immunology 10: 1550.

41. Wang, Q., S. Wei, H. Zhou, G. Shen, X. Gan, S. Zhou, J. Qiu, C. Shi, and L. Lu. 2019. Hyperglycemia exacerbates acetaminophen- induced acute liver injury by promoting liver-resident macrophage proinflammatory response via AMPK/PI3K/AKT-mediated oxidative stress. Cell Death Discovery 5: 119.

42. Xin, C., H. Quan, J.M. Kim, Y.H. Hur, J.Y. Shin, H.B. Bae, and J.I. Choi. 2019. Ginsenoside Rb1 increases macrophage phagocytosis through p38 mitogen-activated protein kinase/Akt pathway. Journal of Ginseng Research 43 (3): 394-401.

43. Yan, L., L. Lu, F. Hu, D. Shetti, and K. Wei. 2019. Piceatannol attenuates RANKL-induced osteoclast differentiation and bone resorption by suppressing MAPK, NF-kappaB and AKT signalling pathways and promotes Caspase3-mediated apoptosis of mature osteoclasts. Royal Society Open Science 6 (6): 190360.

44. Fischer, J., S. Gutierrez, R. Ganesan, C. Calabrese, R. Ranjan, G. Cildir, N. Judith Hos, et al. 2019. Leptin signaling impairs macrophage defenses against Salmonella typhimurium. Proceedings of the National Academy of Sciences of the United States of America 116 (33): 16551-16560.

Publisher's Note Springer Nature remains neutral with regard to jurisdictional claims in published maps and institutional affiliations. 